\title{
Review \\ The Potential Impact of Neuroimaging and Translational Research on the Clinical Management of Lacunar Stroke
}

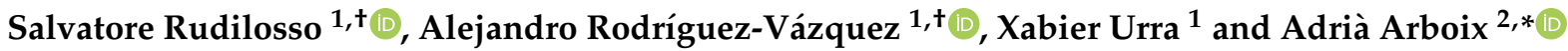 \\ 1 Comprehensive Stroke Center, Hospital Clínic of Barcelona, 08036 Barcelona, Spain; srudilos@clinic.cat (S.R.); \\ alrodriguez@clinic.cat (A.R.-V.); xurra@clinic.cat (X.U.) \\ 2 Cerebrovascular Division, Department of Neurology, Hospital Universitari del Sagrat Cor, \\ Universitat de Barcelona, 08034 Barcelona, Spain \\ * Correspondence: aarboix@quironsalud.es; Tel.: +34-93-494-8940; Fax: +34-93-494-8906 \\ + These authors contributed equally to this work.
}

check for updates

Citation: Rudilosso, S.; Rodríguez-Vázquez, A.; Urra, X.; Arboix, A. The Potential Impact of Neuroimaging and Translational Research on the Clinical Management of Lacunar Stroke. Int. J. Mol. Sci. 2022, 23, 1497. https://doi.org/ $10.3390 /$ ijms23031497

Academic Editor: Paola Rocca

Received: 21 December 2021

Accepted: 24 January 2022

Published: 28 January 2022

Publisher's Note: MDPI stays neutral with regard to jurisdictional claims in published maps and institutional affiliations.

Copyright: (C) 2022 by the authors. Licensee MDPI, Basel, Switzerland. This article is an open access article distributed under the terms and conditions of the Creative Commons Attribution (CC BY) license (https:// creativecommons.org/licenses/by/ $4.0 /$ )

\begin{abstract}
Lacunar infarcts represent one of the most frequent subtypes of ischemic strokes and may represent the first recognizable manifestation of a progressive disease of the small perforating arteries, capillaries, and venules of the brain, defined as cerebral small vessel disease. The pathophysiological mechanisms leading to a perforating artery occlusion are multiple and still not completely defined, due to spatial resolution issues in neuroimaging, sparsity of pathological studies, and lack of valid experimental models. Recent advances in the endovascular treatment of large vessel occlusion may have diverted attention from the management of patients with small vessel occlusions, often excluded from clinical trials of acute therapy and secondary prevention. However, patients with a lacunar stroke benefit from early diagnosis, reperfusion therapy, and secondary prevention measures. In addition, there are new developments in the knowledge of this entity that suggest potential benefits of thrombolysis in an extended time window in selected patients, as well as novel therapeutic approaches targeting different pathophysiological mechanisms involved in small vessel disease. This review offers a comprehensive update in lacunar stroke pathophysiology and clinical perspective for managing lacunar strokes, in light of the latest insights from imaging and translational studies.
\end{abstract}

Keywords: cerebrovascular disease; stroke; ischemic stroke; lacunar stroke; small vessel disease; recent small subcortical infarcts

\section{Introduction: Clinical Relevance and Aims of the Review}

Lacunar ischemic strokes are caused by small infarctions that occur in regions supplied by one perforating artery and represent from 11 to $27 \%$ of acute strokes, according to different series [1]. Lacunar strokes have milder symptoms than strokes, due to large vessel disease, and mortality is exceptional during hospitalization. However, about $20 \%$ of patients who had a lacunar stroke will present a recurrent cerebrovascular event, $25 \%$ will not survive, and 30\% will have some degree of functional dependence at five-year follow-up [2]. About half of the patients with a first-ever lacunar ischemic stroke have mild cognitive impairment of subcortical vascular features, and its presence may be a predictor of subcortical vascular dementia in the medium-long-term [3]. Lacunar strokes are not isolated cerebrovascular events, but often represent the tip of the iceberg of a systemic disease affecting the microcirculation, defined as small vessel disease (SVD), which is considered to be the second cause of dementia, as well as the cause of other severe neuropsychiatric disorders, extrapyramidal symptoms, and frailty in the elderly [4]. Despite the high prevalence of lacunar strokes, and the socio-economic impact related to serious long-term-prognostic implications, no specific SVD treatment is available and most of the treatments do not differ from the management of non-cardioembolic ischemic strokes. Notwithstanding, the development of new imaging techniques, or refinement of existing ones, is providing fruitful insights into the diagnosis and pathophysiology of lacunar strokes [5]. Modeling 
SVD mechanisms and reproducing lacunar strokes in animal models is challenging [6,7], but translational research remains crucial for identifying new therapeutic targets and developing potential therapeutic agents. The review aims to provide a clinical approach to lacunar ischemic stroke management and future direction from imaging and translational evidence. Hence, we addressed how neuroimaging may assist clinicians in diagnosing and managing patients presenting with a lacunar stroke and reviewed the value of new biomarkers and insight from translational studies.

\section{Terminology and Correlations between Histopathological, Clinical and Imaging Definitions}

The terminology adopted to describe small cerebral infarcts, in the territory of perforating arteries, counts tens of different terms that have been used in research and clinical practice [5]. The discrepancies in the terminology and classification of SVD markers are, in part, the result of the integration of terms deriving from anatomical, histopathological, clinical, and radiological fields, which evolved from the first anatomopathological observation, at the end of the 19th century, to the latest neuroimaging techniques capable of assessing single perforating artery morphology and function (Figure 1).

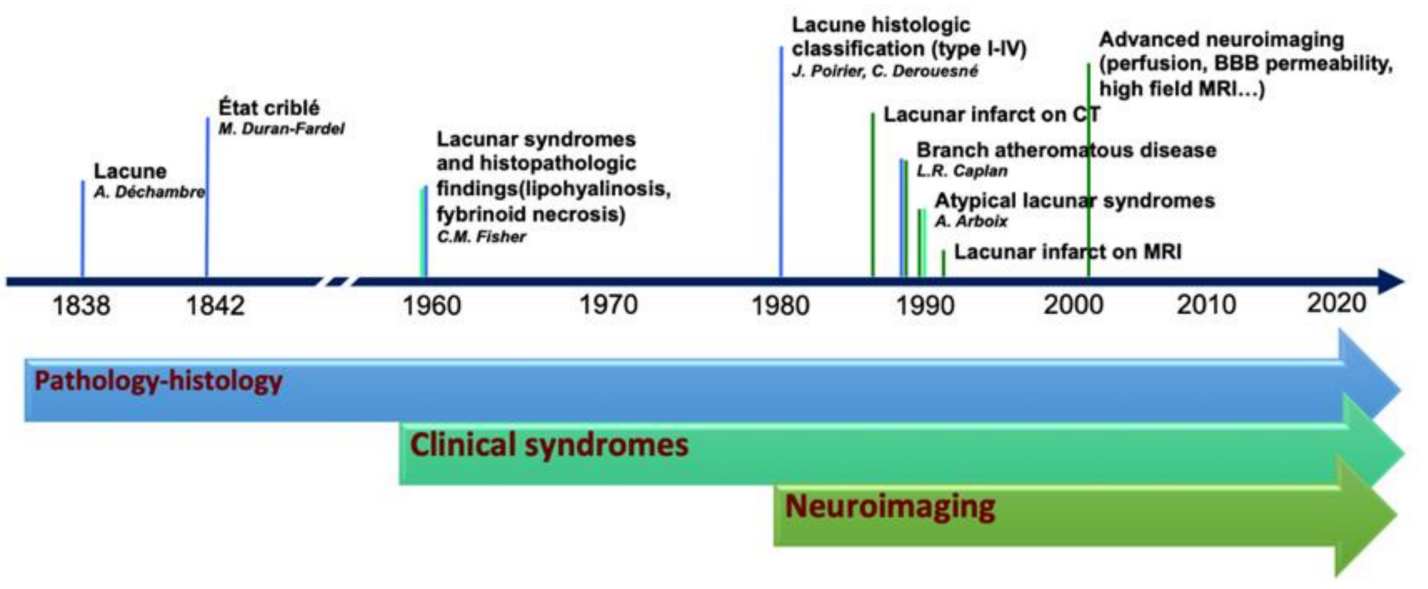

Figure 1. Historical evolution of the knowledge in lacunar strokes.

Lacunar ischemic stroke is a term used to define an acute neurological focal deficit, typically a lacunar syndrome, due to ischemia occurring in a small brain region $(<15 \mathrm{~mm})$ supplied by a single perforating artery, consistently with the lacunar hypothesis of an intrinsic small arteriolar disease [8]. From an etiopathological perspective, a lacunar infarct may appear as a small, incomplete infarction in different stages, from early parenchymal rarefaction and variable inflammatory cells and reactive gliosis (type $2 \mathrm{a}$ ) to complete neuronal loss, spongiosis, and, finally, complete cavitation (type 1a) [9]. The perforating arteries may show typical arteriosclerotic concentric media thickening of the branches smaller than $200 \mu \mathrm{m}$, lipohyalinosis, while the more proximal branches (up to $800 \mu \mathrm{m}$ ) may show microatheromas and microvascular thrombosis $[8,10]$. On neuroimaging, the lacunar infarcts may correspond to small subcortical lesions with an ischemic appearance, on either CT or MRI, while the small perforating arteries are not visible using conventional imaging acquisitions. It is generally assumed that lacunar syndromes represent the clinical manifestation of a perforating artery's occlusion, due to progressive lipohyalinosis and superposed microthrombosis, while neuroimaging provides an in vivo surrogate of the small infarction in the brain. Nevertheless, the correlation between clinical syndromes, as well as radiological and histological findings, is not absolute (Figure 2). For example, the identification, on imaging, of an acute cortical stroke necessarily excludes the SVD etiology of the stroke, even if the patient presented with a classical lacunar syndrome. On the other hand, a small subcortical infarct on imaging might be caused by mechanisms other than arteriosclerotic SVD, such as an embolism or large vessel atherosclerotic plaques 
occluding perforating artery branches. Lacunar infarcts and lacunes may be clinically silent or misrecognized, while disabling lacunar syndromes may be produced by infarcts so small that they might be missed, even when using techniques with high spatial definition, such as an MRI. Histopathological findings include small infarctions in the brain parenchyma, associated with typical lesions of the perforating vessels, such as lipohyalinosis, arteriolar disorganization, and perivascular edema $[8,11]$. However, many years might separate the stroke event from the post-mortem study, and clinical-histological correlations might be challenging to establish. Lacunes are small cavities filled by cerebrospinal fluid (CSF) in the subcortical white matter or gray matter structures that might represent the final ischemic stage after a perforating artery occlusion but are also difficult to differentiate from enlarged perivascular spaces in imaging studies.

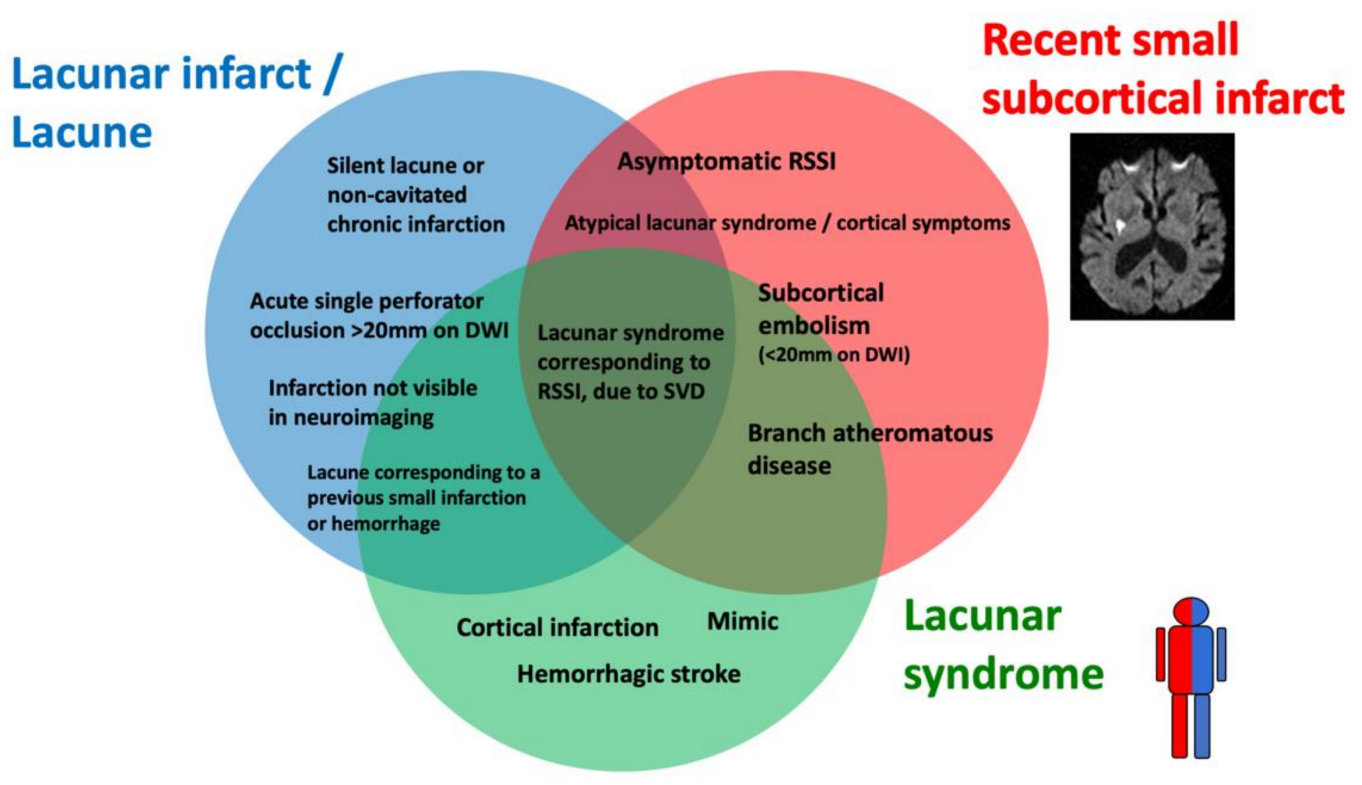

Figure 2. Histological-clinical-radiological correlations in lacunar strokes.

The terminology and definitions used to classify different types of strokes, as well as the different features of SVD, need to be homogenous in research to simplify systematic search strategies and improve the external validity of observational studies and randomized clinical trials (RCTs). Several clinical classifications exist, based on clinical and radiological criteria, to differentiate lacunar strokes from strokes with other etiology. The etiology of stroke subtype may be defined according to different classifications that are based on clinical and radiological criteria. In the Trial of Org 10172 in Acute Stroke Treatment (TOAST) [12], a stroke due to small artery occlusion (lacunar) should present with a traditional lacunar syndrome and have no lesion on imaging, or a subcortical ischemic lesion smaller than $15 \mathrm{~mm}$, in the absence of a mayor cardioembolic source or arterial stenosis $>50 \%$ on vascular imaging. The atherosclerosis, SVD, cardiac pathology, other causes, dissection classification (ASCOD) [13], and causative classification system for acute ischemic stroke classification (CCS) [14] define the grades of likelihood for SVD etiology. According to the ASCOD definition, the probability of lacunar stroke etiology depends on the presence of a compatible lesion on imaging and other radiological signatures of SVD, such as lacunes or white matter lesions. According to the CCS classification, the probability of lacunar stroke etiology (evident, probable, or possible) is based on the evidence of a consistent lesion on imaging and lacunar syndrome presentation in the absence of alternative causative mechanisms. The standards for reporting vascular changes of neuroimaging (STRIVE) classification was proposed, in order to standardize the different features of SVD in neuroimaging, rather than differentiating lacunar strokes from other strokes subtypes. According to this classification, mostly based on MRI, the result of an occlusion of one perforating artery is classifiable as recent small subcortical infarct (RSSI), 
white matter hyperintensity (WMH), or lacune, according to clinical-radiological criteria (Figure 3).

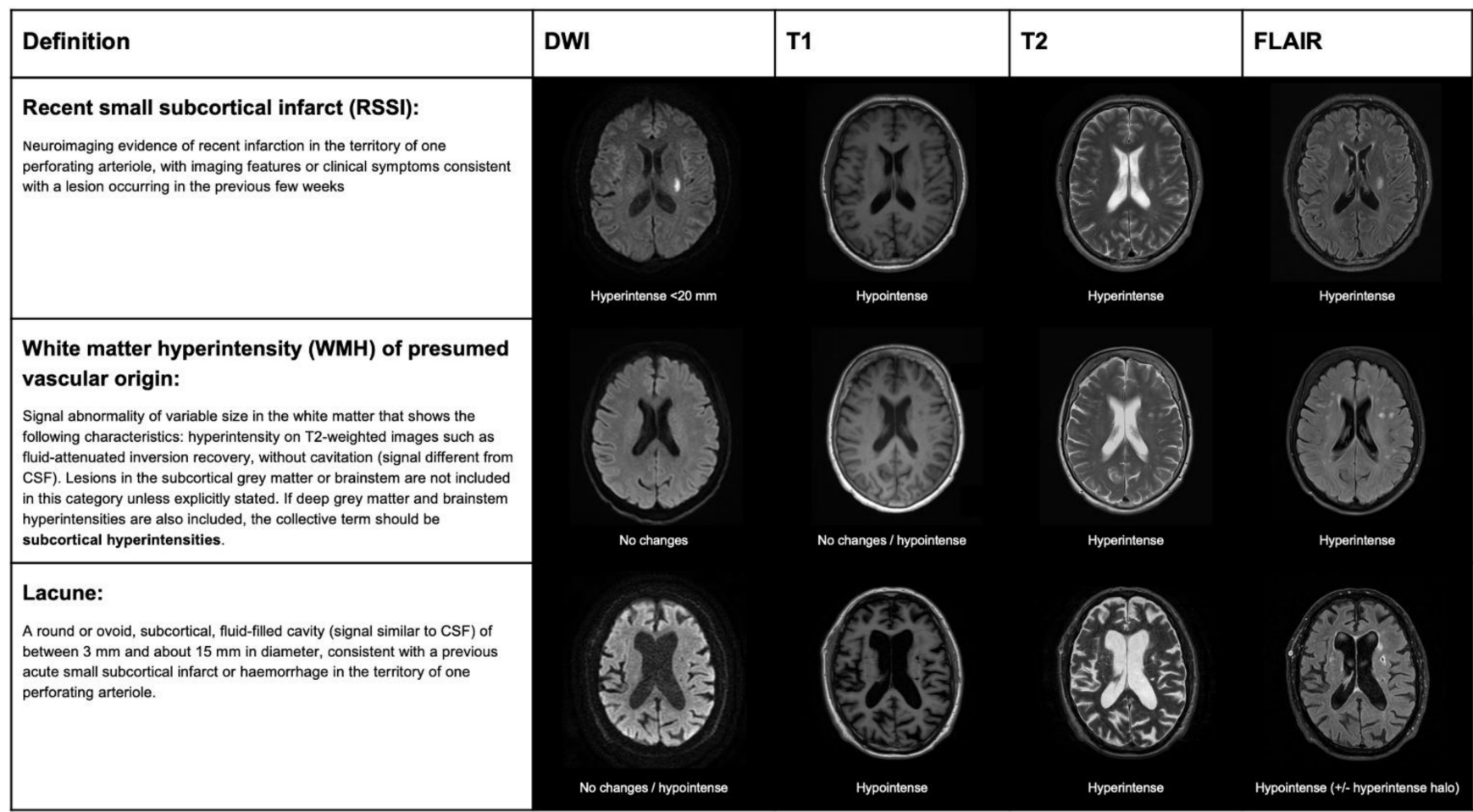

Figure 3. STandards for ReportIng Vascular changes on nEuroimaging (STRIVE) classification for ischemic lesions on MRI, produced by lacunar stroke.

The histopathological features of lacunar infarcts were described by Fisher [8], and a classification for the different appearance of these infarcts, depending on the stage or presence of hemorrhagic features, was proposed by Derouesné and Poirer [9]. However, there is still no consensus for the terminology and reporting of SVD features in histology and their correspondence with imaging features [11,15].

In this review, we used the term RSSI to refer to neuroimaging evidence, according to the STRIVE definition. We also maintained the term lacunar stroke or syndrome, as appropriated, exclusively to refer to clinical events (i.e., acute management context). In contrast, lacunar infarction (non-cavitated lesion, either acute or chronic) and lacune (chronic cavitated lesion $<15 \mathrm{~mm}$ ) were preferred for pathological findings or assumptions.

\section{Mechanisms of Lacunar Strokes: From Pathology Studies to Advanced Neuroimaging}

Lacunar ischemic strokes are highly associated with hypertensive arteriosclerosis and other vascular risk factors [16-18]. However, about 15-30\% of patients with lacunar ischemic strokes had no history of hypertension, suggesting that other vascular risk factors, including aging, and complex mechanisms affecting the microvascular function might play a significant role in the pathogenesis of lacunar stroke. Some of these potential mechanisms that may contribute to perforating artery occlusion, and how they might represent a possible target for therapeutic interventions, are summarized in Table 1. 
Table 1. Possible mechanisms involved in lacunar stroke pathogenesis.

\begin{tabular}{|c|c|c|c|c|}
\hline Mechanism & Description & Evidence & Unsolved Issues & Possible Intervention Target \\
\hline $\begin{array}{l}\text { Hypertensive } \\
\text { arteriosclerosis }\end{array}$ & $\begin{array}{c}\text { Progressive } \\
\text { hypertensive-related } \\
\text { arteriosclerotic injury. } \\
\text { Superposed } \\
\text { microthrombosis may } \\
\text { lead to complete } \\
\text { arteriolar occlusion. }\end{array}$ & $\begin{array}{l}\text { Typical histopathological } \\
\text { findings in } \\
\text { perforating arteries. } \\
\text { Indirect evidence from } \\
\text { high field MRI } \\
\text { techniques }[19,20] .\end{array}$ & $\begin{array}{l}\text { Non hypertensive patients } \\
\text { may also present with lacunar } \\
\text { stroke [21]. In vivo } \\
\text { radiological confirmation of } \\
\text { small artery wall alterations } \\
\text { are not available. }\end{array}$ & $\begin{array}{l}\text { Hypertension is the most } \\
\text { modifiable risk factor for } \\
\text { stroke secondary prevention } \\
\text { [22]. In patients with lacunar } \\
\text { strokes, intensive vs. standard } \\
\text { blood pressure reduction did } \\
\text { not reduce the risk of all stroke } \\
\text { recurrency, although it } \\
\text { reduced the risk of intracranial } \\
\text { hemorrhage (SPS3) [23]. }\end{array}$ \\
\hline $\begin{array}{l}\text { Atherosclerosis } \\
\text { (branch } \\
\text { atheromatous } \\
\text { disease) }\end{array}$ & $\begin{array}{l}\text { Atherosclerotic plaques } \\
\text { in the main cerebral } \\
\text { vessel may occlude the } \\
\text { orifice of perforating } \\
\text { arterioles }[24,25] .\end{array}$ & $\begin{array}{l}\text { Anatomopathological } \\
\text { studies [18]. Small plaques } \\
\text { are also visible using high } \\
\text { field MRI techniques for } \\
\text { vessel wall } \\
\text { assessment [24]. }\end{array}$ & $\begin{array}{l}\text { Atherosclerosis in large vessel } \\
\text { arteries may represent } \\
\text { an epiphenomenon. }\end{array}$ & $\begin{array}{l}\text { Lipid lowering is effective for } \\
\text { reducing stroke recurrence in } \\
\text { non-cardioembolic strokes } \\
\text { (SPARCL trial) [26]. Other new } \\
\text { drugs aimed to stabilize the } \\
\text { inflammatory process in } \\
\text { atherosclerosis, which might } \\
\text { represent a promising } \\
\text { therapeutic target. }\end{array}$ \\
\hline
\end{tabular}
lacunar strokes may be patent in pathology

Small emboli, either from proximal atherosclerotic plaques or cardiac source, may produce single or multiple small subcortical infarcts.

Increased blood flow on

CT perfusion suggests

recanalization of an

embolic occlusion of a studies [8] and advanced

7T MRI techniques [27].

perforating artery [28]

Subcortical infarcts in

animal models produced

by microembolism [6].

\section{Chronic hypoperfusion} of distal vascular territories may lead to

Chronic global cerebral

hypoperfusion progressive ischemia in the white matter. Small infarctions may occur in the edges of $\mathrm{WMH}$ and contribute to

SVD progression.
In animal models, small subcortical infarcts may be produced by bilateral carotid occlusions [6].
There is an association between atrial fibrillation, load of subcortical infarcts, and WMH [29], but direct evidence of embolism is lacking.

Multiple RSSIs do not exclude mechanisms related to SVD (about $20 \%$ of RSSI present multiple infarcts, especially in patients with severe SVD [30]).
Treatments aimed to stabilize active plaques or anticoagulant treatment, in case of mayor embolic source. Prothrombotic state (i.e., acute cancer), marantic, or infectious endocarditis should be ruled out in patients with multiple subcortical strokes.
The causal relationship between hypoperfusion and

SVD progression in

longitudinal studies is controversial [31],

as hypoperfusion might also be also secondary to reduced metabolism in WMH.
Vasodilatory drugs to increase brain perfusion: mononitrate isosorbide, nitric oxide. (LACI-2) [32].
Some studies on post-mortem brain samples did not confirm the association of markers of endothelial dysfunction or BBB leakage and SVD [37,38].

A causal relationship with focal BBB leakage prior lacunar strokes is to be determined. BBB permeability variations are mild and difficult to measure in SVD. Blood markers of endothelial dysfunction and inflammation are are not specific of lacunar stroke subtype [39].
Anti-inflammatory drugs: colchicine in non-cardioembolic strokes (CONVINCE) [40], uric acid

(URICO-ICTUS) [41], and canakinumab [42]. impaired coupling

between metabolic

demand and

nutrient supply. compared to cortical strokes [36]. 
Table 1. Cont.

\begin{tabular}{|c|c|c|c|c|}
\hline Mechanism & Description & Evidence & Unsolved Issues & Possible Intervention Target \\
\hline $\begin{array}{l}\text { Focal } \\
\text { hypoperfusion and } \\
\text { compensatory blood } \\
\text { flow in acute } \\
\text { perforating artery } \\
\text { occlusion }\end{array}$ & $\begin{array}{l}\text { Abrupt reduction in } \\
\text { blood flow after } \\
\text { perforating artery } \\
\text { occlusion, regardless the } \\
\text { causing mechanisms } \\
\text { (either intrinsic SVD or } \\
\text { atheroembolic). The } \\
\text { extent and the time to } \\
\text { establish infarction may } \\
\text { depend on factors such } \\
\text { as compensatory blood } \\
\text { flow through capillary } \\
\text { network and } \\
\text { cerebrovascular reserve. }\end{array}$ & $\begin{array}{l}\text { Perfusion studies show } \\
\text { persistence of residual } \\
\text { blood flow, in the territory } \\
\text { of perforating arteries } \\
\text { corresponding to } \\
\text { RSSI [43,44]. Sequential } \\
\text { imaging from row } \\
\text { perfusion sequences may } \\
\text { show retrograde flow, } \\
\text { suggesting collateral } \\
\text { circulation involvement in } \\
\text { RSSI [28,45,46] } \\
\text { Microscopic studies } \\
\text { showed a dense capillary } \\
\text { network, linking } \\
\text { contiguous perforating } \\
\text { arteries and few arteriolar } \\
\text { anastomoses [47]. }\end{array}$ & $\begin{array}{l}\text { Lack of direct evidence of } \\
\text { perforating artery occlusion } \\
\text { and recruiting collateral } \\
\text { circulation in RSSI }\end{array}$ & $\begin{array}{l}\text { Thrombolysis in lacunar stroke } \\
\text { would not be effective without } \\
\text { compensatory mechanisms } \\
\text { maintaining the tissue viable } \\
\text { until recanalization. Perfusion } \\
\text { imaging-based thrombolysis, } \\
\text { outside of the conventional } \\
\text { time window, may also be } \\
\text { effective in patients with RSSI. } \\
\text { Vasodilatory agents may } \\
\text { improve collateral recruitment. } \\
\text { Neuroprotective agents may } \\
\text { reach the ischemic area } \\
\text { through retrograde in the } \\
\text { territory supplied by an } \\
\text { occluded perforating artery. }\end{array}$ \\
\hline
\end{tabular}

BBB: blood-brain barrier; CONVINCE: colchicine for prevention of vascular inflammation in non-cardioembolic stroke; LACI-2: lacunar intervention trial-2; RSSI: recent small subcortical infarcts; SVD: small vessel disease; SPARCL: stroke prevention by aggressive reduction in cholesterol levels; SPS3: secondary prevention of small subcortical stroke trial; URICO-ICTUS: uric acid in patients with acute stroke trial; WMH: white matter hyperintensities.

Lacunar infarcts are one of the distinctive markers of SVD, including both sporadic [16] and monogenic SVD types (such as cerebral autosomal dominant arteriopathy with subcortical infarcts and leukoencephalopathy (CADASIL) [48-50]) and other rare hereditary diseases [51]. In histopathological studies of lacunar infarcts, the perforating arteries typically show thickening of the media, lipohyalinosis, segmental arterial disorganization, and fibrinoid degeneration [18]. However, the histopathological findings represent the end stages of the disease, and the correlations with the clinical event are difficult to establish in most cases. For example, in the pathology studies conducted by Fisher in 114 patients with lacunar infarcts, the perforating arteries supplying the lacunar infarct/lacune had no vascular occlusion, in only $10 \%$ of the cases, and only 4 patients had no history of hypertension [8]. A recent systematic review of 39 pathology studies, including more than 4000 lacunar infarcts, highlighted that occlusion of the supplying perforating arteries was found only in a minority of cases, and the prevalence of hypertension was lower, although still higher than $50 \%$ of patients [11]. This discordancy could result from therapeutic advances in stroke prevention, such as hypertensive treatment, which could reduce the likelihood of presenting severe progressive stenosis of perforating arteries, while other alternative mechanisms, including embolic occlusions, might have prevailed. However, these mechanistic interpretations are drawn from evidence obtained in post-mortem studies conducted, in most cases months or years after the clinic event, when many factors, including remodeling of the microvasculature, changes in blood flow, and antithrombotic therapies, may have altered the structural features of the small vessels.

Neuroimaging of lacunar stroke has the main advantage of shortening the time from symptoms onset to examination, providing imaging with high temporospatial accuracy and offering insights on the mechanisms occurring during infarction and on the course over time in longitudinal studies. However, imaging evidence has to be considered indirect in most cases, especially in SVD studies, as the small branches of perforating vessel are not visible, not even using high-definition MRI (although the main branches may be visualized using high resolution MRI angiography at 7T [27]). Much information provided by imaging studies is difficult to generalize and depends on many factors, including different criteria to define markers in imaging, acquisition protocols, processing software, and interobserver reliability. Therefore, new markers in imaging research studies need to be tested through various steps of validation. In brief, a newly discovered marker needs a proof of concept (does the marker measure a specific change related to a disease process?) and proof of 
principle (discriminates cases vs. controls, severity, or prognosis). The technique to assess the marker should be repeatable (precision under the same operating conditions) and reproducible (precision under different operating conditions). Finally, the marker should be effective as an endpoint for clinical studies (i.e., surrogate of a clinical endpoint) and costeffective for use in large multicentre clinical trials. However, only a few markers in imaging used in SVD research meet most of these validation criteria. More detailed information on the validation of imaging markers in SVD is available in the harmonizing brain imaging methods for vascular contributions to neurodegeneration (HARNESS) position paper [52].

Deep perforating arteries are currently considered end-terminal vascular territories that supply subcortical white matter and deep grey structures. According to this classical theory, the occlusion of a perforating artery would irreversibly lead to infarction of the whole tissue, supplied by an occluded perforating artery within a few minutes after occlusion [53]. However, about $20 \%$ of patients with a lacunar stroke presented a transient ischemic attack in the previous hours or days [54], and patients presenting with a lacunar syndrome may recover without showing any ischemic lesion on brain imaging [55]. Perfusion studies on CT and MRI showed that small areas of hypoperfusion (but not a complete absence of it) are visible in some patients with a confirmed RSSI on follow-up imaging, including areas of potentially viable tissue (ischemic penumbra) [43,44], in contrast with the hypothesis of a complete flow obstruction, without compensation in a terminal arterial territory. The capsular warning syndrome was first described in patients with lacunar stroke involving the internal capsule presenting repeated, stereotyped episodes of motor lacunar syndrome or sensorimotor lacunar syndrome, within 24-72 h, with complete recovery between episodes, which involved two of three body parts (face, arm, or leg), or more, without cortical symptoms [56,57]. Other studies described lacunar strokes with a similar clinical stuttering course, in other anatomical regions as the pons [58,59]. Several mechanistic interpretations of the capsular warning syndrome have been formulated, including hemodynamic failure in the presence of a stenotic perforating artery, arteriolar vasospasm, and peri-infarct depolarization $[56,60]$. However, the fluctuating insufficiency of a residual blood flow compensation through collateral vessels from nearby perforating arteries would also be consistent with the clinical stuttering presentation. Although perforating artery branches are not directly visible in vivo using conventional imaging, three perfusion studies, one based on CT perfusion [28] and two on MRI perfusion [45,46], provided similar results of indirect evidence of hemodynamical compensation, through retrograde blood flow filling centripetally the ischemic regions that evolved into a RSSI, suggesting the presence of microscopic collateral supply by a capillary network. In few cases, the ischemic area showed an early and anterograde filling, corresponding to normal or increased perfusion, indicating the patency of the perforating artery during image acquisition, consistent with recanalization, suggesting an embolic origin of the occlusion in a minority of patients [28].

Although small perforating vessels are not directly visible on conventional MRI, small thrombi might be spotted as blooming artifacts on gradient-echo or SWI sequences in the pathway of a perforating artery in less than $20 \%$ of patients with a RSSI [61]. However, this technique probably has low sensitivity (no gold standard is available in imaging), and specificity could be hampered by badly impaired blood-brain barrier (BBB) permeability and hemosiderin deposits. Other non-conventional techniques based on high-resolution MRI for vessel wall imaging enable the detection of non-stenotic atherosclerotic plaques occluding emerging perforating arteries, despite apparently normal vascular imaging $[19,62]$. The number and morphology of the perforating arteries are also evaluable using high field MRI [63]. However, the long acquisition time and sensitivity to movement artifacts limit the feasibility of these techniques in patients with acute strokes.

In the last 20 years, many authors focused their attention on vascular and endothelial dysfunction in SVD using imaging to assess vascular function measures for BBB permeability, blood flow, and vascular stiffness [4]. The endothelial dysfunction seems to have a crucial role in the pathogenesis of SVD, involving vascular inflammatory mechanisms altering the $\mathrm{BBB}$ permeability and extravasation of inflammatory particles into the ex- 
tracellular space, causing perivascular edema and microglial dysfunction. Endothelial inflammation may increase pro-thrombotic activity, favoring microthrombosis, and affect small vessel autoregulation and capillary heterogeneity [4]. Early studies, assessing BBB permeability using dynamic contrast-enhanced MRI, showed that patients with lacunar strokes have increased BBB permeability, compared to patients with cortical strokes [64]. Other measures of vascular function as cerebrovascular reactivity appeared to be impaired in patients with SVD [65] and lacunar strokes [66] in a few cross-sectional studies. However, it is difficult to obtain these measures prior to the appearance of new subcortical infarcts in longitudinal studies, due to the relatively low rates of incident strokes and use of secondary prevention measures.

The radiological fate of RSSI is variable and hardly predictable, as one-third of RSSI evolve into a lacune, while others may disappear or leave a non-cavitated lesion $[67,68]$. BBB leakage into CSF appeared to be a predicting factor of cavitation in one longitudinal study [69], but further longitudinal studies might identify other risk factors. The clinical and prognostic relevance of RSSI cavitation remains to be determined, although some associations have been found with severe progression of SVD and cognitive impairment [70]. Nevertheless, most lacunes might not be related to prior clinical events and represent accidental findings on neuroimaging studies. The spatial correlation with motor and sensitive pathways is highly related to overt clinical symptoms [71], but some lacunes, even in eloquent areas, might not have been recognized clinically. Therefore, some lacunes might result from progressive injury and cavitation, which could be less clinically evident. For example, new cavities usually appear silently in the edges of white matter hyperintensities in patients with CADASIL [72], and lacunes are associated with deep medullary vein stiffening and occlusion, due to venous collagenosis [73].

\section{Plasmatic Biomarkers in Patients with Lacunar Strokes}

The main pathophysiological mechanisms, such as coagulation/fibrinolysis processes, endothelial dysfunction, and inflammation, involved in cerebral SVD may be assessed by measuring specific plasma biomarkers [34,38]. Patients with lacunar strokes present a pro-thrombotic state, demonstrated by overall increased plasma levels of tissue plasminogen activator (TPA), plasminogen activator inhibitor (PAI), fibrinogen, and D-dimer, compared to patients without stroke, but lower levels, compared to other stroke subtypes, characterized by larger infarct volumes [38,74]. Similarly, endothelial activation markers (homocysteine and von Willebrand factor) and leukocyte adhesion molecules (E-selectin, P-selectin, ICAM-1, and VCAM-1) expression is augmented in patients with lacunar stroke, compared to healthy controls, but not different from other stroke subtypes [38,74]. Several plasma markers of inflammation have been assessed in patients with SVD and lacunar strokes [38]. C-reactive protein (CRP) is a sensitive, but not specific, marker of systemic inflammation that has been related to stroke risk in the community-dwelling population [75] and risk of ischemic recurrence in patients who had a lacunar stroke [76,77]. Other blood markers of inflammation, such as TNF- $\alpha$ and IL-6, have been related to ischemic recurrence in patients with lacunar stroke [78]. However, the levels of most inflammatory markers were similar, or even lower, in patients with lacunar stroke, compared to other stroke subtypes, suggesting that most of these markers are due to the impact of the acute ischemic injury and may depend more on the stroke volume, rather than the different etiology [39]. Table 2 summarizes the potential biomarkers of lacunar strokes that have been previously studied, grouped by their primary related pathophysiological mechanism. 
Table 2. Plasma biomarkers in lacunar stroke.

\begin{tabular}{|c|c|c|c|}
\hline Mechanism & Molecule & Findings & References \\
\hline \multirow{4}{*}{$\begin{array}{l}\text { Coagulation and } \\
\text { fibrinolysis }\end{array}$} & $\begin{array}{l}\text { Tissue plasminogen } \\
\text { activator (TPA) }\end{array}$ & $\begin{array}{l}\text { - Higher in lacunar stroke vs. non-stroke, } \\
\text { acutely and chronically } \\
\text { - Similar in lacunar stroke vs. non-lacunar } \\
\text { stroke, acutely and chronically }\end{array}$ & $\begin{array}{l}\text { Lindgren, } 1996 \text { [79] } \\
\text { Salobir, } 2003 \text { [80] } \\
\text { Jood, } 2005 \text { [81] }\end{array}$ \\
\hline & $\begin{array}{l}\text { Plasminogen activator } \\
\text { inhibitor (PAI) }\end{array}$ & $\begin{array}{l}\text { - Higher in lacunar stroke vs. non-stroke, } \\
\text { acutely and chronically } \\
\text { - } \quad \text { Similar in lacunar stroke vs. non-lacunar } \\
\text { stroke, acutely and chronically }\end{array}$ & $\begin{array}{l}\text { Lindgren, } 1996[79] \\
\text { Salobir, } 2002 \text { [80] } \\
\text { Jood, } 2005 \text { [81] } \\
\text { Yokokawa, 2008 [82] } \\
\text { Ilhan, } 2010 \text { [83] }\end{array}$ \\
\hline & Fibrinogen & $\begin{array}{l}\text { - } \quad \text { Similar in lacunar stroke vs. } \\
\text { non-stroke, acutely } \\
\text { - } \quad \text { Higher in lacunar stroke vs. } \\
\text { non-stroke, chronically } \\
\text { - } \quad \text { Lower in lacunar stroke vs. non-lacunar } \\
\text { stroke, acutely and chronically }\end{array}$ & $\begin{array}{l}\text { Kilpatrick, } 1993 \text { [84] } \\
\text { Beamer, } 1995 \text { [85] } \\
\text { Bath, 1998 [86] } \\
\text { Kataoka, } 2000 \text { [87] } \\
\text { Salobir, } 2003 \text { [80] } \\
\text { Jood, } 2008 \text { [81] } \\
\text { Álvarez-Pérez, 2011 [88] } \\
\text { Beer, 2011 [89] } \\
\text { Zhang, 2011 [90] } \\
\text { Datta, 2014 [91] }\end{array}$ \\
\hline & D-dimer & $\begin{array}{l}\text { - Higher in lacunar stroke vs. non-stroke, } \\
\text { acutely and chronically } \\
\text { - } \quad \text { Lower in lacunar stroke vs. non-lacunar } \\
\text { stroke, acutely and chronically }\end{array}$ & $\begin{array}{l}\text { Takano, } 1992 \text { [92] } \\
\text { Kataoka, } 2000 \text { [87] } \\
\text { Ajeno, 2002 [93] } \\
\text { Salobir, } 2003 \text { [80] } \\
\text { Ilhan, } 2010 \text { [83] } \\
\text { Montaner, 2008 [94] } \\
\text { Brouns, 2009 [95] } \\
\text { Isenegger, } 2010 \text { [96] } \\
\text { Álvarez-Pérez, 2011 [88] }\end{array}$ \\
\hline \multirow{4}{*}{$\begin{array}{l}\text { Endothelial } \\
\text { dysfunction }\end{array}$} & Homocysteine & $\begin{array}{l}\text { - Higher in lacunar stroke vs. non-stroke, } \\
\text { acutely and chronically } \\
\text { - Similar in lacunar stroke vs. non-lacunar } \\
\text { stroke, acutely }\end{array}$ & $\begin{array}{l}\text { Eikelboom, } 2000 \text { [97] } \\
\text { Hassan, } 2004 \text { [98] } \\
\text { Parnetti, } 2004 \text { [99] } \\
\text { Khan, } 2007 \text { [100] } \\
\text { Khan, } 2008 \text { [101] } \\
\text { Yokokawa, 2008 [82] } \\
\text { Beer, 2011 [89] } \\
\text { Jeong, 2011 [102] } \\
\text { Pavlovic, } 2011 \text { [103] } \\
\text { Lavallée, } 2013 \text { [104] }\end{array}$ \\
\hline & $\begin{array}{l}\text { Von Willebrand } \\
\text { factor (vWF) }\end{array}$ & $\begin{array}{l}\text { - } \quad \text { Higher in lacunar stroke vs. } \\
\text { non-stroke, acutely } \\
\text { - } \quad \text { Lower in lacunar stroke vs. non-lacunar } \\
\text { stroke, acutely }\end{array}$ & $\begin{array}{l}\text { Beer, } 2011 \text { [89] } \\
\text { Hanson, } 2011 \text { [105] } \\
\text { Lavallée, } 2013 \text { [104] }\end{array}$ \\
\hline & E-selectin & 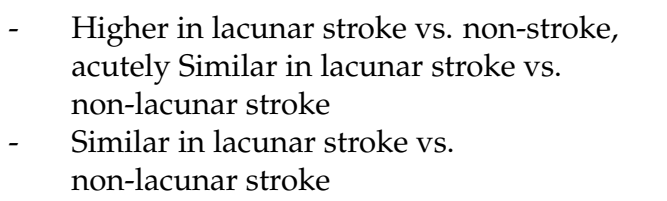 & $\begin{array}{l}\text { Kozuka, } 2002 \text { [106] } \\
\text { Beer, 2011 [89] }\end{array}$ \\
\hline & P-selectin & $\begin{array}{l}\text { - Higher in lacunar stroke vs. non-stroke but } \\
\text { only in some studies } \\
\text { - } \quad \text { Similar in lacunar stroke vs. } \\
\text { non-lacunar stroke }\end{array}$ & $\begin{array}{l}\text { Bath, } 1998 \text { [86] } \\
\text { Kozuka, } 2002 \text { [106] } \\
\text { Tsai, 2009 [107] } \\
\text { Ilhan, } 2010 \text { [83] } \\
\text { Turgut, } 2011 \text { [108] } \\
\text { Lavallée, } 2013 \text { [104] }\end{array}$ \\
\hline
\end{tabular}


Table 2. Cont.

\begin{tabular}{|c|c|c|c|}
\hline Mechanism & Molecule & Findings & References \\
\hline & $\begin{array}{l}\text { Intercellular adhesion } \\
\text { molecule } 1 \text { (ICAM-1) }\end{array}$ & $\begin{array}{l}\text { - Higher in lacunar stroke vs. non-stroke, } \\
\text { acute and chronically } \\
\text { - Similar in lacunar stroke vs. non-lacunar } \\
\text { stroke, acutely }\end{array}$ & $\begin{array}{l}\text { Castellanos, } 2002 \text { [109] } \\
\text { Hassan, } 2003 \text { [98] } \\
\text { Supanc, } 2011 \text { [110] } \\
\text { Rouhl, } 2012 \text { [111] }\end{array}$ \\
\hline & $\begin{array}{l}\text { Vascular cellular } \\
\text { adhesion molecule } 1 \\
\text { (VCAM-1) }\end{array}$ & $\begin{array}{l}\text { - Similar in lacunar stroke vs. non-lacunar } \\
\text { stroke, acutely }\end{array}$ & $\begin{array}{l}\text { Supanc, } 2011[110] \\
\text { Rouhl, } 2012 \text { [111] } \\
\text { Brwon, } 2015[112]\end{array}$ \\
\hline \multirow{3}{*}{ Inflammation } & $\begin{array}{l}\text { C-reactive protein } \\
(\mathrm{CRP})\end{array}$ & $\begin{array}{l}\text { - Higher in lacunar stroke vs. non-stroke, } \\
\text { acutely and chronically } \\
\text { - Similar in lacunar stroke vs. non-lacunar } \\
\text { stroke, acutely and chronically }\end{array}$ & $\begin{array}{l}\text { Ladenvall, } 2006 \text { [81] } \\
\text { Yokokawa, } 2008 \text { [82] } \\
\text { Montaner, } 2008 \text { [94] } \\
\text { Nakase, 2008 [113] } \\
\text { Álvarez-Pérez, 2011 [88] } \\
\text { Beer, 2011 [89] } \\
\text { Turgut, } 2011 \text { [108] } \\
\text { Mitaki, 2016 [114] }\end{array}$ \\
\hline & $\begin{array}{l}\text { Tumor necrosis } \\
\text { factor } \alpha(\mathrm{TNF} \alpha)\end{array}$ & $\begin{array}{l}\text { - Higher in lacunar stroke vs. } \\
\text { non-stroke, acutely }\end{array}$ & $\begin{array}{l}\text { Castellanos, } 2002 \text { [109] } \\
\text { Domac, } 2007 \text { [115] } \\
\text { Boehme, } 2016[78]\end{array}$ \\
\hline & Interleukin 6 (IL-6) & $\begin{array}{l}\text { - Higher in lacunar stroke vs. non-stroke, } \\
\text { acutely } \\
\text { - } \quad \text { Lower in lacunar stroke vs. non-lacunar } \\
\text { stroke, acutely }\end{array}$ & $\begin{array}{l}\text { Beamer, } 1995 \text { [85] } \\
\text { Vila, } 2000 \text { [116] } \\
\text { Castellanos, 2002 [109] } \\
\text { Salobir, } 2004 \text { [117] } \\
\text { Domac, } 2007 \text { [115] } \\
\text { Guldiken, } 2008 \text { [118] } \\
\text { Boehme, } 2016 \text { [78] }\end{array}$ \\
\hline
\end{tabular}

\section{Insights from Translational Research}

Direct evidence on SVD and small lacunar infarcts pathophysiology is particularly challenging to obtain from post-mortem studies and in neuroimaging for the low mortality and limited spatial definition, respectively. Therefore, experimental models of lacunar strokes are warranted to broaden the knowledge on the pathophysiological mechanisms and identify new therapeutic targets. However, several technical and conceptual issues may limit their translatability to humans [6,7]. First, there are relevant anatomical differences between animal models and the human brain, such as the ratio of gray/white matter and vessel distribution and density. There are different techniques to obtain small subcortical ischemic lesions, modeling lacunar infarcts, that include lesions produced by different mechanisms not consistent with SVD pathophysiology (i.e., vasoconstriction [119-122] and microembolism [123-125]), surgical or chemical endothelium injury leading to perforating artery occlusion $[126,127]$, hypoperfusion produced by surgical bilateral common carotid artery occlusion [128,129], and others produced spontaneously in animals reproducing SVD model, such as hypertensive animals $[6,130,131]$ or models of monogenic SVD (i.e., CADASIL) [132-134]. While the induced focal ischemic techniques are more reproducible, the spontaneous ones are difficult to trace or know the exact beginning. The main advantages and flaws of each technique are summarized in Table 3. 
Table 3. Experimental models of stroke modeling lacunar infarcts.

\begin{tabular}{|c|c|c|c|c|c|}
\hline Mechanism & Techniques & Description & Advantages & Disadvantages & References \\
\hline $\begin{array}{l}\text { Vasoconstriction of } \\
\text { perforating arteries }\end{array}$ & $\begin{array}{c}\text { Endothelin-1, nitric oxide } \\
\text { synthase inhibitor, and } \\
\text { L-NAME }\end{array}$ & $\begin{array}{l}\text { Strong vasoconstrictive } \\
\text { action that affects } \\
\text { several microvessels }\end{array}$ & $\begin{array}{l}\text { Small subcortical } \\
\text { infarcts }\end{array}$ & $\begin{array}{l}\text { Multiple vessels } \\
\text { affected at once }\end{array}$ & $\begin{array}{l}\text { Horie, } 2008 \text { [119] } \\
\text { Capone, } 2011 \text { [120] } \\
\text { Cipolla, } 2013 \text { [135] } \\
\text { Cui, 2013 [122] }\end{array}$ \\
\hline Embolism & $\begin{array}{l}\text { Microspheres, } \\
\text { microthrombi injection, } \\
\text { atheroemboli, black beads, } \\
\text { preformed clots, and } \\
\text { silicone rubber cylinders }\end{array}$ & $\begin{array}{l}\text { Injection of different } \\
\text { materials in the carotid } \\
\text { to produce } \\
\text { micro-occlusions by } \\
\text { lodging in brain vessels }\end{array}$ & $\begin{array}{l}\text { Multiple } \\
\text { subcortical } \\
\text { infarcts }\end{array}$ & $\begin{array}{l}\text { Mostly cortical } \\
\text { infarcts, } \\
\text { mechanism not } \\
\text { related to SVD }\end{array}$ & $\begin{array}{l}\text { Rapp, } 2003 \text { [123] } \\
\text { Wang, } 2012[124] \\
\text { Silasi, } 2015 \text { [125] }\end{array}$ \\
\hline Spontaneous lesions & $\begin{array}{l}\text { High salt, spontaneous } \\
\text { in SHRSP, surgical } \\
\text { narrowing of the aorta, and } \\
\text { genetic mutations in the } \\
\text { renin-angiotensin system }\end{array}$ & $\begin{array}{l}\text { Mice breeds with an } \\
\text { increased risk of stroke, } \\
\text { genetically or } \\
\text { surgically induced }\end{array}$ & $\begin{array}{l}\text { Mechanism } \\
\text { consistent with } \\
\text { hypertensive } \\
\text { SVD }\end{array}$ & $\begin{array}{l}\text { Difficult to track } \\
\text { lesion location } \\
\text { and timing }\end{array}$ & $\begin{array}{l}\text { Hainsworth, } 2008 \text { [130] } \\
\text { Bailey, } 2009 \text { [6] } \\
\text { Mencl, } 2013 \text { [131] }\end{array}$ \\
\hline $\begin{array}{l}\text { Perforating artery } \\
\text { occlusion }\end{array}$ & $\begin{array}{l}\text { Surgery (pial vessel } \\
\text { disruption model), } \\
\text { sodium laurate } \\
\text { thrombosis, and } \\
\text { photothrombosis }\end{array}$ & $\begin{array}{l}\text { Endothelium damage } \\
\text { and thrombosis, using } \\
\text { toxic substances or } \\
\text { surgical models }\end{array}$ & $\begin{array}{l}\text { Accurate } \\
\text { localization of } \\
\text { the lesions }\end{array}$ & $\begin{array}{l}\text { Strokes larger } \\
\text { than lacunar } \\
\text { infarcts }\end{array}$ & $\begin{array}{l}\text { Walz, } 2017 \text { [126] } \\
\text { Wen, } 2019 \text { [127] }\end{array}$ \\
\hline $\begin{array}{l}\text { Transient large } \\
\text { vessel occlusion }\end{array}$ & $\begin{array}{l}\text { Bilateral common carotid } \\
\text { artery occlusion }\end{array}$ & $\begin{array}{l}\text { Repeated transient large } \\
\text { vessel occlusion, } \\
\text { followed by reperfusion }\end{array}$ & $\begin{array}{l}\text { Mechanism } \\
\text { reflects the } \\
\text { hypoperfusion } \\
\text { in SVD }\end{array}$ & $\begin{array}{l}\text { Lesions are not } \\
\text { related to small } \\
\text { vessel pathology }\end{array}$ & $\begin{array}{l}\text { Choi, } 2018 \text { [128] } \\
\text { Kwon, } 2015 \text { [129] }\end{array}$ \\
\hline Genetic models & $\begin{array}{l}\text { CADASIL mouse models, } \\
\text { COL4A1/2 mouse models }\end{array}$ & $\begin{array}{l}\text { Studies in mice with rare } \\
\text { genetic disorders which } \\
\text { make them prone } \\
\text { to SVD }\end{array}$ & $\begin{array}{l}\text { Mechanism } \\
\text { related to genetic } \\
\text { SVD etiology }\end{array}$ & $\begin{array}{l}\text { Not enough } \\
\text { brain affection }\end{array}$ & $\begin{array}{c}\text { Ayata, } 2010 \text { [132] } \\
\text { Joutel, } 2011 \text { [133] } \\
\text { Ruchoux, } 2003 \text { [134] }\end{array}$ \\
\hline
\end{tabular}

CADASIL: cerebral autosomal dominant arteriopathy with subcortical infarcts and leukoencephalopathy COL4A1/2: collagen type 4 alpha 1 chain gene; L-NAME: N-nitro-L-arginine methylester; SHRSP: spontaneously hypertensive stroke-prone rats; SVD: small vessel disease.

\section{Prevalence and Diagnostic/Topographic Accuracy of the Lacunar Syndromes}

In the 1960s, C.M. Fisher described the histopathological correlates of four clinical syndromes (pure motor, pure sensitive, ataxic-hemiparesis, and clumsy-hand dysarthria), defined as lacunar syndromes $[136,137]$. Some years later, J.P. Mohr et al. also described the sensorimotor syndrome corresponding to capsular-thalamic infarctions [138]. The sensorimotor syndrome represents about $20-30 \%$ of the lacunar syndromes, but it is also the one with the highest likelihood $(>30 \%)$ of being caused by non-lacunar strokes (i.e., cortical strokes). The pure motor is the most prevalent lacunar syndrome (50-70\%) but had a low specificity for spatial location (any point of the central motor pathway) $[136,139]$, while the pure sensitive represents about $10-20 \%$ of lacunar syndromes $[139,140]$ and have high specificity for ventroposterolateral thalamic infarctions. Dysarthria-clumsy hand syndrome $[139,141]$ and ataxic hemiparesis [142-144] represent about 3-6\% of lacunar syndromes and may correspond to RSSI, mainly in the internal capsule and pons, with few cases $(<7 \%)$ corresponding to cortical strokes [145]. Other atypical syndromes have been described, including monoparesis, partial cortical, or extrapyramidal symptoms, as well as deficits due to cranial nerve nuclei involvement in brainstem infarcts [8,146]. Moreover, up to $30 \%$ of cortical strokes may also present with motor or sensitive symptoms alone mimicking subcortical infarcts. The diagnostic accuracy of the lacunar syndromes for RSSI has been addressed in several studies, most of them using MRI as the gold standard for lesion location, as detailed in Table 4. 
Table 4. Lacunar syndrome: correspondence with imaging findings.

\begin{tabular}{|c|c|c|c|c|c|c|c|c|}
\hline Reference & Cohort & $\begin{array}{c}\text { Lacunar } \\
\text { Syndrome, } \\
n(\%)\end{array}$ & $\begin{array}{l}\text { Cortical } \\
\text { Syndrome, } \\
n(\%)\end{array}$ & $\begin{array}{c}\text { RSSI/ } \\
\text { NonLacunar } \\
\text { Syndrome, } \\
n(\%)\end{array}$ & $\begin{array}{l}\text { Non-RSSI/ } \\
\text { Lacunar } \\
\text { Syndrome, } \\
n(\%)\end{array}$ & $\begin{array}{c}\text { Lacunar } \\
\text { Syndrome/DWI } \\
\text { Negative, } n(\%)\end{array}$ & $\begin{array}{c}\text { Cortical } \\
\text { Syndrome/DWI } \\
\text { Negative, }(\%)\end{array}$ & $\begin{array}{c}\text { Lacunar } \\
\text { Syndrome } \\
\text { Positive } \\
\text { Predictive } \\
\text { Value }\end{array}$ \\
\hline $\begin{array}{c}\text { Potter, } 2010 \\
\text { [147] }\end{array}$ & 313 & $79(25)$ & $136(43)$ & $21 / 93(23)$ & $7 / 44$ (16) & $35 / 79(44)$ & $43 / 136(32)$ & $\begin{array}{l}46 \% \%^{\circ} \\
84 \%\end{array}$ \\
\hline $\begin{array}{c}\text { Ay, } 1999 \\
{[148]}\end{array}$ & 62 & $62(100)$ & - & - & $10 / 62(16)$ & $9 / 62(14)$ & - & $68 \%$ \\
\hline $\begin{array}{c}\text { Stapf, } 2000 \\
{[149]}\end{array}$ & 54 & $54(100)$ & - & - & $3 / 54(6)$ & $0 / 54(0)$ & - & $94 \%$ \\
\hline $\begin{array}{l}\text { Lindgren, } \\
2000 \text { [150] }\end{array}$ & 23 & $23(100)$ & - & - & $2 / 23(8)$ & $1 / 23(4)$ & - & $86 \%$ \\
\hline $\begin{array}{c}\text { Seifert, } 2005 \\
\text { [151] }\end{array}$ & 93 & $41(44)$ & $\begin{array}{l}\text { POCS (39) } \\
\text { PACS (15) } \\
\text { TACS (1) }\end{array}$ & 15/93 (16) & - & - & - & $\begin{array}{l}44 \%{ }^{\wedge} \\
83 \%{ }^{\wedge}\end{array}$ \\
\hline $\begin{array}{c}\text { Wessels, } \\
2005 \text { [152] }\end{array}$ & 73 & $73(100)$ & - & - & $30 / 73(38)$ & $0 / 73(0)$ & - & $58 \%$ \\
\hline $\begin{array}{c}\text { Arboix } \\
2010 *[153]\end{array}$ & 879 & 879 (100) & - & - & $146 / 879$ (17) & - & - & $83 \%$ \\
\hline $\begin{array}{l}\text { Altmann, } \\
2014 \text { [154] }\end{array}$ & 119 & $119(100)$ & - & - & $16 / 119(13)$ & $17 / 86(20)$ & - & $\begin{array}{l}60 \% \\
65 \% \\
\end{array}$ \\
\hline $\begin{array}{l}\text { Giacomozzi, } \\
2019 \text { [155] }\end{array}$ & 1796 & $478(26)$ & $1313(74)$ & $346 / 1313(26)$ & $104 / 478(21)$ & - & - & $78 \%$ \\
\hline $\begin{array}{l}\text { Arba, } 2020 \\
* *[156]\end{array}$ & 568 & $330(58)$ & $238(42)$ & $59 / 238(25)$ & $102 / 330(31)$ & - & - & $\begin{array}{l}25 \% \text { *** } \\
75 \% \text { **** }\end{array}$ \\
\hline
\end{tabular}

* Diagnostic imaging was MRI (41\%) and CT (59\%); ** CT scan as follow-up imaging; *** worst-case scenario (negative CT scan considered as non-RSSI); ${ }^{* * *}$ best-case scenario (negative CT scan considered as RSSI). Adding NIHSS $<7$ to lacunar syndrome improved the positive predictive value to $97 \%$ for both worst- and best-case scenarios. ${ }^{\circ}$ Worst-case scenario (negative DWI considered as non-RSSI). ${ }^{\circ}$ Values only considering DWI-confirmed stroke. ^Values including only LACS. ^ Values including both LACS and POCS. · Values including both MRI and CT follow-up imaging. .. Values including only MRI follow-up imaging. LACS: lacunar syndrome; POCS: posterior occlusion circulation syndrome; TACS: total anterior circulation syndrome. Modified from Potter et al., 2010 [147].

\section{Neuroimaging in Patients with Suspected Lacunar Stroke}

The confirmation of a RSSI in patients presenting with a lacunar syndrome requires identification on neuroimaging (either CT or MRI) of a lesion consistent with a small ischemic stroke in the territory of a single deep perforating artery (lenticulostriate, thalamoperforating, thalamogeniculate, paramedian, and deep medullary arteries), corresponding to subcortical white matter (centrum semiovale and corona radiate, internal and external capsule, and short and long fibers in the brainstem) or deep grey structures (basal ganglia, thalamus, and nuclei) in the brainstem. CT was the first technique capable of identifying small focal hypoattenuations consistent with lacunar strokes [157]. Small subcortical infarcts are hardly visible as early small ischemic changes in the first hours after symptom onset on CT scans and difficult to distinguish from older lesions in patients with SVD. The introduction of MRI for stroke assessment made possible a more precise topographic and morphologic RSSI characterization [158]. In particular, the implementation of DWI was crucial to identify recent lesions as hyperintensities (few minutes from stroke onset and remaining visible for about 3-5 weeks), while older lesions are visible in the other structural sequences $[159,160]$. Despite the high diagnostic accuracy of MRI, small lesions might be missed, depending on several factors, such as the magnetic field, correction of motion artifacts, and width of the consecutive slice acquisition [148]. Thus, the absence of hyperintense lesions on DWI should not rule out a lacunar stroke in the presence of a lacunar syndrome. Finally, the presence of a RSSI on MRI could represent the residual infarction of a larger perfusion deficit involving more than a perforating artery or large vessel territories, as shown in some perfusion studies [161]. In other cases, perfusion 
deficits in the territory of one perforating artery may be reversible [55]. A stroke results from dynamic ischemic processes, depending on several factors, such as metabolic demand, collateral blood supply, time of ischemia, and reperfusion. The snapshot provided by imaging studies might provide incomplete information that needs to be complemented by clinical assessment and appropriate complementary exams. Even so, the etiological diagnosis must be considered presumptive in lacunar stroke in the absence of a pathological confirmation and, especially, cautious in case of incomplete or inconclusive workup or when possible concomitant causes are present [162].

Identifying RSSI is particularly challenging in the acute stroke assessment for technical limitations of standard imaging techniques. However, imaging may assist clinicians in the early presumptive diagnostic orientation for assessing reperfusion in eligible patients, secondary prevention strategies, and the clinical interpretation of threatening clinical courses, such as neurological deterioration and stuttering symptoms in lacunar strokes. The most commonly adopted techniques for acute stroke assessment are based on CT imaging, while MRI is used in fewer centers and might be less practical and more timeconsuming in the emergency service. Although the accuracy of non-contrast CT evaluation is extremely low in the acute phase, multimodal imaging, including CT angiography and perfusion maps, may improve the detection of small perfusion deficits corresponding to lacunar stroke. Several studies confirmed the utility of CT perfusion in lacunar stroke assessment in the past few years (Table 5), despite some important limitations, due to low sensitivity for very small infarcts and those located in the brainstem and lack of external validation and automatic detection tools. In addition, sensitivity and specificity are not comparable between studies, due to different population, selection criteria, RSSI prevalence, and study design. For example, some of the studies had extremely high specificity, probably due to pre-evaluation selection of patients or strict criteria to identify a perfusion deficit [163-166]. Nevertheless, the accuracy of non-contrast CT is highly improved by perfusion maps $[43,163,164,166]$, which should be carefully evaluated for the presence of focal perfusion alterations, consistent with the clinical presentation.

Table 5. Studies assessing the diagnostic accuracy of CT perfusion in patients with lacunar stroke or confirmed RSSI.

\begin{tabular}{|c|c|c|c|}
\hline Reference & Population & Perfusion Maps & Main Findings \\
\hline Rudilosso, 2015 [43] & $\begin{array}{l}\text { A total of } 33 \text { patients with lacunar } \\
\text { syndrome (16 lacunar strokes, } \\
13 \text { non-lacunar strokes, and } 4 \text { no } \\
\text { ischemic lesions). Lacunar stroke } \\
\text { defined as infarct volume }<1.767 \mathrm{~cm}^{3} \\
\text { (the volume of a sphere with a } \\
\text { diameter } 1.5 \mathrm{~cm} \text { ) on DWI. }\end{array}$ & $\begin{array}{l}\text { Postprocessing software: CT Neuro } \\
\text { Perfusion Syngo.via (Siemens } \\
\text { Healthcare GmbH) for visual } \\
\text { assessment. MIStar (Apollo Medical } \\
\text { Imaging Technology, Melbourne, } \\
\text { Australia) for core/penumbra } \\
\text { threshold analysis. } \\
\text { Perfusion maps: CBF, CBV, MTT, } \\
\text { Tmax, TTP, TTD, and MIP. }\end{array}$ & $\begin{array}{l}\text { SE and PPV for lacunar stroke higher } \\
\text { than non-contrast CT ( } 63 \% \text { vs. } 19 \%) \text {. } \\
\text { CTP was more sensitive for } \\
\text { supratentorial lesions, compared with } \\
\text { infratentorial lesions ( } 65 \% \text { versus } \\
16 \%) \text { SP was low }(20 \%) \text { and } \\
\text { influenced by low lacunar stroke } \\
\text { prevalence. TTD was the most } \\
\text { informative map for the identification } \\
\text { of ischemic lesions. }\end{array}$ \\
\hline Das, 2015 [163] & $\begin{array}{l}\text { A total of } 88 \text { patients with lacunar } \\
\text { syndrome (after excluding stroke } \\
\text { mimics). RSSI: } 59 / 88(67 \%) \text {. }\end{array}$ & $\begin{array}{c}\text { Postprocessing software: } \\
\text { GE Healthcare } \\
\text { Perfusion maps: CBV, CBF, and MTT. }\end{array}$ & $\begin{array}{l}\text { SE56\%, SP 83\%. CTP increased the } \\
\text { diagnostic performance 5-fold over } \\
\text { non-contrast CT. MTT were the most } \\
\text { informative maps to identify RSSI. }\end{array}$ \\
\hline Benson, 2016 [164] & $\begin{array}{l}\text { A total of } 113 \text { patients: } 37 \text { with } \\
\text { ischemic lesions on DWI }<20 \mathrm{~mm} \text { in } \\
\text { maximum diameter (either cortical or } \\
\text { subcortical) and } 76 \text { without ischemic } \\
\text { lesions. Ischemic lesions }>20 \mathrm{~mm} \text {, and } \\
\text { patients treated with iv tPA were } \\
\text { excluded from the analysis. }\end{array}$ & $\begin{array}{l}\text { Postprocessing software: Vitrea } \\
\text { workstation (Vital Imaged, } \\
\text { Minnetonka, Minnesota) } \\
\text { Perfusion maps: TTP, MTT, CBV, } \\
\text { and CBF. }\end{array}$ & $\begin{array}{l}\text { TTP were the maps with highest SE } \\
(49 \%) \text {, and lowest for non-contrast CT } \\
(3 \%) \text {. SP was high regardless the map } \\
\text { evaluated (all }>97 \%) \text {. The perfusion } \\
\text { lesions on CTP appeared larger than } \\
\text { the lesion on DWI. }\end{array}$ \\
\hline
\end{tabular}


Table 5. Cont.

\begin{tabular}{|c|c|c|c|}
\hline Reference & Population & Perfusion Maps & Main Findings \\
\hline Tan, 2016 [161] & $\begin{array}{l}\text { A total of } 182 \text { patients with ischemic } \\
\text { strokes ( } 31 \text { single subcortical, } \\
9 \text { multiple subcortical, } 34 \text { cortical only, } \\
33 \text { non-confluent cortical-subcortical, } \\
\text { and } 75 \text { confluent cortical-subcortical). }\end{array}$ & $\begin{array}{c}\text { Postprocessing software: Advantage } \\
\text { Windows (GE Medical Systems) and } \\
\text { Extended Brilliance Workspace } \\
\text { (Philips Healthcare, Best, Netherlands) } \\
\text { Perfusion maps: MTT. }\end{array}$ & $\begin{array}{l}39 \% \text { of the RSSI (single subcortical) on } \\
\text { DWI had a perfusion deficit. However, } \\
\text { for } 67 \% \text { of them, the perfusion deficit } \\
\text { was larger than the DWI lesion and } \\
\text { were associated with a large vessel } \\
\text { occlusion on CT angiography. }\end{array}$ \\
\hline Cao, 2016 [165] & $\begin{array}{l}\text { A total of } 62 \text { patients: } 32 \text { with RSSI } \\
\text { and } 30 \text { without lesions on DWI. }\end{array}$ & $\begin{array}{l}\text { Postprocessing software: RAPID } \\
\text { iSchemicView (Menlo Park, CA, US) } \\
\text { Perfusion maps: CBF, CBV, MTT, } \\
\text { and Tmax. }\end{array}$ & $\begin{array}{l}\text { MTT showed } 56 \% \text { SE. No false } \\
\text { positive perfusion images were rated. }\end{array}$ \\
\hline $\begin{array}{l}\text { García-Esperón, } \\
2021 \text { [166] }\end{array}$ & $\begin{array}{c}\text { A total of } 106 \text { patients with lacunar } \\
\text { syndrome: RSSI, } 33 \text { cortical and } \\
14 \text { posterior fossa strokes. Patients } \\
\text { without lesions on DWI } \\
\text { were excluded. }\end{array}$ & $\begin{array}{c}\text { Postprocessing software: MIStar } \\
\text { (Apollo Medical Imaging Technology, } \\
\text { Melbourne, Australia) } \\
\text { Perfusion maps: CBF, CBV, MTT, } \\
\text { and DT. }\end{array}$ & $\begin{array}{c}42 \% \text { SE, } 80 \% \text { SP for RSSI. Visual } \\
\text { inspection of CTP maps had higher SE } \\
\text { than the automated method } \\
(42 \% \text { vs. } 6 \%) \text {. Sensitivity on } \\
\text { non-contrast CT was very low }(<4 \%) \text {. }\end{array}$ \\
\hline \multicolumn{4}{|c|}{$\begin{array}{l}\text { CBF: cerebral blood flow; CBV: cerebral blood volume; CTP: CT perfusion; DT: delay time; DWI: diffusion } \\
\text { weighted imaging; MIP: maximum intensity projection; MTT: mean transient time; PPV: positive predictive value } \\
\text { RSSI: recent small subcortical infarct; SE: sensitivity; SP: specificity; Tmax: time to maximum; TTD: time to drain } \\
\text { TTP: time to peak. }\end{array}$} \\
\hline
\end{tabular}

\section{Lacunar Stroke Management}

No disease-specific treatment targeting intrinsic SVD mechanisms currently exists. Therefore, patients with lacunar stroke should receive the treatments that have demonstrated their efficacy in the acute stroke treatment and secondary prevention, such as systemic thrombolysis and antithrombotic therapies, respectively, to avoid irreversible damage in the brain.

\section{Intravenous Thrombolysis}

Some concerns have been raised about the efficacy of this therapy in lacunar strokes, since the underlying pathophysiology might be related to mechanisms alternative to thrombosis in small vessel disease. However, the main RCTs that proved the efficacy of systemic thrombolysis in patients with stroke included patients with lacunar syndromes and showed no effect modification by the presumed vascular subtype [167]. In terms of safety, the risk of haemorrhagic complications after systemic thrombolysis in patients with lacunar infarcts is lower than in patients with other stroke etiologies; additional observational studies supported the benefit of this treatment in all stroke subtypes $[168,169]$. Therefore, intravenous thrombolysis is indicated for patients presenting with a lacunar syndrome in the first $4.5 \mathrm{~h}$ from symptom onset, indifferently of other stroke types [170]. Recent studies demonstrated that systemic thrombolysis is also effective in the extended time window (more than $4.5 \mathrm{~h}$ from stroke-onset) in patients with stroke viable tissue, quantified with MRI (DWI-FLAIR mismatch) and CT perfusion [171]. The MRI-based thrombolysis in the wake-up stroke trial (WAKE-UP), based on DWI/FLAIR mismatch in acute MRI evaluation, included patients with lacunar strokes, showing a benefit from IV thrombolysis, similar to large vessel strokes in a post hoc analysis [172]. Other studies (extending the time for thrombolysis in emergency neurological deficits trial, ECASS IV-ExTEND), based on penumbra quantification using CT perfusion, demonstrated the efficacy of IV thrombolysis in stroke with large volume, thus excluding patients with small subcortical infarcts [173]. Future studies should assess which perfusion maps are most accurate for selecting patients with small perfusion deficits for reperfusion in the extended time window, including those with mild symptoms and focal hypoperfusion, in the territory of a perforating artery that might be at risk of neurological deterioration [174]. 


\section{Secondary Prevention \\ 10.1. Antplatelets}

Any single antiplatelet therapy is effective for the secondary prevention of ischemic strokes of almost all subtypes, including lacunar ischemic strokes [175]. Dual antiplatelet therapy (DAPT) has been assessed for secondary prevention of ischemic stroke, including lacunar strokes. The SPS3 trial showed that acetyl-salicylic acid (ASA) plus clopidogrel in patients with lacunar stroke in the prior six months did not reduce the incidence of new strokes but increased the risk of bleeding and death [176]. However, later trials demonstrated the efficacy of short-term DAPT started early (12-24 h) and maintained for 21-90 days, compared with single antiplatelet therapy in patients with mild strokes and TIA. The clopidogrel in high-risk patients with acute non-disabling cerebrovascular events (CHANCE) [177] and platelet-oriented inhibition in new transient ischemic attack and minor ischemic stroke (POINT) [178] trials assessed the efficacy of ASA plus clopidogrel vs. ASA alone in patients with NIHSS $<3$ and $<5$, randomized within 24 and $12 \mathrm{~h}$ from stroke onset, during 21 and 90 days, respectively. More recently, the acute stroke or transient ischemic attack treated with ticagrelor or ASA for prevention of stroke and death trial (THALES) [179] assessed the efficacy of ticagrelor plus ASA in patients with NIHSS < 5, randomized within $24 \mathrm{~h}$, during 30 days. In these RCTs, DAPT vs. ASA alone decreased the incidence of new strokes and cardiovascular events, despite minor increases in haemorrhagic risk, which was higher in studies with longer DAPT treatment. Nevertheless, few data are available in the subgroups of patients with lacunar strokes in these RCTs. A post hoc analysis of the CHANCE trial showed that patients with multiple strokes had lower stroke recurrences than those with a single stroke (about half of them were classified as lacunar strokes), which benefited more from DAPT vs. ASA alone [180]. Some observational studies showed that DAPT is safe in patients with lacunar infarct and early neurological deterioration, even in those who received IV tPA, and seemed to improve the functional outcomes $[174,181,182]$. In the SPS3 trial, patients treated with DAPT had a risk of major bleeding, compared to ASA alone, but this difference was due to extracranial hemorrhage and mainly occurred after one year of DAPT. Therefore, DAPT is not indicated in patients with a history of prior lacunar stroke. However, DAPT might be the best option for early secondary prevention in patients with mild lacunar stroke for a short period, as recommended by the American and European Guidelines [170,183]. Moreover, DAPT might limit the effect of antiplatelet resistance, measured by impedance aggregometry, to either ASA or clopidogrel in mild strokes, especially in patients with SVD [184,185]. Patients with a lacunar stroke presenting with high NIHSS might also benefit from DAPT, since they have a relatively small infarct volume, compared to cortical strokes with similar clinical severity and theoretically lower risk of haemorrhagic transformation. Imaging selection, according to stroke volume and other SVD factors that increase the risk of intracranial bleeding (i.e., the presence of multiple cerebral microbleeds on MRI), should be evaluated in the future in patients who could benefit from DAPT.

Cilostazol is a phosphodiesterase-3 inhibitor commonly used in Asiatic countries for stroke prevention with mild antiplatelet effect and other properties on endothelial dysfunction, myelin repair, neuroprotection, and inflammation. A recent meta-analysis of RCTs, comparing cilostazol vs. ASA, clopidogrel, or placebo, suggested that cilostazol is effective for stroke prevention, without increasing the hemorrhagic risk [186]. The efficacy appeared higher when treatment duration was longer than six months and in trials including more than $40 \%$ of lacunar stroke type.

The best management of secondary prevention antithrombotic drugs in patients with lacunar stroke is still debated, and further RCTs are needed. At the moment, strong antiplatelet prevention with DAPT, instead of single antiplatelet therapy, should be considered only for mild strokes for the first few weeks after acute stroke, when the ischemic recurrence risk is higher. Future trials are warranted to confirm whether adding cilostazol to aspirin or clopidogrel for long-term prevention could offer additional protective effects, due to non-platelet activity, without increasing the hemorrhagic risk. 
Despite the efficacy of antiplatelet therapy in preventing new strokes, lacunar infarcts represent only the tip of the iceberg of SVD. The progression of the disease and appearance of related serious conditions, such as cognitive impairment, extrapyramidal syndromes, and psychiatric disorders, might require other treatments targeting alternative mechanisms in SVD.

\subsection{Statins}

The perforating arteries supplying the territory of a lacunar infarct may present arteriosclerosis with microatheromas in the proximal portions or branch atheromatous disease occluding the perforators' origin [18] and benefit from lipid-lowering. The SPARCL trial demonstrated that, in patients with a stroke or transient ischaemic attack and LDL > 100, $80 \mathrm{mg}$ of atorvastatin daily reduces the risk of new cerebrovascular events, despite a mild increase in haemorrhagic strokes. A post hoc analysis, according to stroke subtypes, showed that patients with large and small vessel occlusions benefited similarly from statin use [187], reassuring some of the concerns rose about the possible increased haemorrhagic risk in patients with SVD. However, there is no consensus about the target for LDL reduction in these patients.

\subsection{Anti-Hypertensive Treatments}

The hypertension-induced mechanisms in SVD are complex and include structural and functional maladaptation to high blood pressure and arterial pulsatility, oxidative stress, endothelial dysfunction, BBB disruption, capillary rarefaction, and impaired neurovascular coupling [188]. The effect of hypertension might not be the same in deep perforating and cortical perforating arteries. The former are more exposed to hypertensive injury, due to the high pressure gradient from the main (proximal) cerebral arteries, while the latter (deep medullary arteries penetrating the supratentorial white matter) depends on the (distal) leptomeningeal circulation, with a low pressure gradient, and might be more prone to hypoperfusion [189].

Avoiding hypertension is indicated in the secondary prevention of patients having a stroke $[170,190]$. However, the optimal blood pressure target in patients with lacunar stroke is unknown. In the SPS3 trial, intensive blood pressure treatment (systolic $<130 \mathrm{mmHg}$ ) in patients with lacunar infarcts was not superior to achieving standard blood pressure target (130-150 mmHg) to reduce stroke recurrence, despite a positive trend in favor of intensive blood pressure treatment [23]. Notably, the incidence of stroke recurrences in SPS3 was low in both arms, suggesting that a comprehensive management of patients with lacunar infarcts is advantageous, leaving small room for further benefit attributable to intensive blood pressure lowering. On the other hand, inducing hypotension in patients with SVD could affect the compensatory mechanism in the presence of altered arteriolar stiffness and autoregulation. The potentially harmful effect of intensive blood lowering could also be more relevant in the hyperacute phase for the potential decrease in residual compensatory perfusion flow that could accelerate the ischemic process. However, a substudy of the enhanced control of hypertension and thrombolysis stroke study (ENCHANTED) did not suggest any deleterious effect of acute blood pressure lowering, as the functional outcome in patients with lacunar stroke treated with thrombolysis was similar, independently of standard (blood pressure < 185/110 mmHg) vs. intensive (systolic target 130-140 mmHg) within one hour from randomization [191].

\subsection{Lifestyle Interventions}

Lifestyle interventions include a range of behaviorally modifiable risk factors, such as smoking, physical activity, and diet (i.e, low salt intake), that probably represent the targets with the most significant potential impact in the prevention of all subtypes of stroke $[192,193]$. However, few studies were conducted in the specific population of patients with a lacunar stroke. A small RCT $(n=71)$ in patients with lacunar stroke did not show differences in cardiorespiratory fitness measures in patients randomized to high-intensity 
interval training or moderate-intensity continuous training [194]. Nevertheless, the clear evidence from prior studies, including all subtypes of strokes, encouraging physical activity after stroke can be extrapolated for the lacunar subtype.

\section{New Molecular Targets in Lacunar Stroke}

The current management for patients presenting with a lacunar stroke is based on reperfusion treatment with thrombolytic agents and secondary antithrombotic prevention (antiplatelets), as well as other interventions on modifiable vascular risk factors (i.e., lifestyle intervention, statins, and antihypertensive drugs). However, no specific treatment for SVD exists, and new possible therapeutic targets, involving different mechanisms in SVD, are currently under investigation (Table 1).

As aforementioned, cilostazol is a promising drug in SVD for non-antiplatelet activity on BBB integrity, vasodilation, inflammation, and neuroprotection. A small RCT trial, conducted in Korea (effect of cilostazol in acute lacunar infarction, based on pulsatility index of transcranial doppler, ECLIPse), showed that adding cilostazol to aspirin in the secondary prevention of patients with lacunar stroke decreased intracranial pulsatility in the main cerebral arteries, confirming the pleiotropic effect of cilostazol beyond the antiplatelet activity [195]. The LACI-1 was a phase IIa trial that indicated the safety and tolerability of cilostazol and isosorbide mononitrate (an oxid nitric donor commonly used in angina) in patients with a lacunar stroke, also showing an improvement of vascular function in the white matter measured using cerebrovascular reactivity in blood oxygenation level dependent (BOLD)-MRI. A phase IIb trial (LACI-2) to assess the feasibility, tolerability, and provide clinical outcomes is ongoing [32].

Inflammation represents a potential target in stroke secondary prevention that could particularly fit with SVD etiology, since mechanisms other than thrombosis seem to have a pivotal role outside the acute stroke phase. The URICO-ICTUS trial showed promising results on the efficacy of a potent endogenous antioxidant molecule, such as uric acid, associated with systemic thrombolysis in acute ischemic stroke patients [41]. Although uric acid was not significantly superior to the placebo in the primary outcome measure (excellent outcome defined as modified Rankin Score 0-1 at 90-day follow-up, adjusted risk ratio 1.23 [95\% CI 0.96-1.56]; $p=0.099$ ), it seemed to be particularly effective in some subgroups of patients, such as women, patients with high glucose levels, and milder strokes (NIHSS < 10). However, only $5 \%$ of the patients enrolled had a lacunar stroke, and the efficacy of uric acid in this particular subgroup of stroke should be tested in future trials. Colchicine is an anti-inflammatory drug with different mechanisms of action, including microtubule disruption, neutrophile targeting, and inhibition of inflammasome-mediated IL-1 $\beta$ maturation [196]. The CONVINCE is a phase III PROBE design trial that will assess the efficacy of colchicine in patients with non-severe, non-cardioembolic stroke or high-risk TIA [40]. Canakinumab is a therapeutic monoclonal antibody targeting interleukin-1 $\beta$ innate immunity that reduced cardiovascular events in patients with myocardial infarction and high CRP levels. However, the efficacy in the stroke population has not been assessed yet [42].

\section{Future Directions}

Systemic thrombolysis in patients with lacunar stroke attending later than $4.5 \mathrm{~h}$ from symptom onset is not a marginal issue. It often occurs in patients with stuttering and progressively worsening symptoms. However, the thrombolysis indication in this subgroup of patients is still uncertain, despite the encouraging results from the WAKE-UP trial [172]. Perfusion studies in the acute stage could also identify patients with mild non-disabling symptoms but an evident, relatively large perfusion deficit in the territory of the perforating artery, which may present neurological deterioration and possibly benefit from thrombolysis. While MRI is not feasible in the acute stroke assessment in most stroke centers, automatic CT perfusion software packages are not helpful for detecting small perfusion deficits, and visual assessment could be challenging, especially for untrained readers. 
Therefore, multicentric studies, pooling large series of patients with lacunar strokes, are warranted to improve the automatic detection of small perfusion alterations and validate the results obtained in smaller, single-center cohorts.

Secondary prevention measures, mainly based on antiplatelet and vascular risk factors control, are not much different from other non-cardioembolic ischemic strokes. However, long-term DAPT is hazardous in patients with SVD for increasing the bleeding risk, while the risk of late stroke recurrences and SVD progression is higher in patients with lacunar strokes than in those with large vessel stroke. For these reasons, the research of new drugs targeting non-thrombotic mechanisms of SVD is particularly encouraging for the long-term, secondary prevention of lacunar stroke.

\section{Limitations of the Review}

This review is to be considered a broad overview on several pathophysiological, clinical, diagnostic, and therapeutic issues concerning lacunar stoke. Therefore, the main limitation of the review is the lack of a systematic literature search methodology. However, the main aim of the review is to provide a personal view for clinicians and highlight opportunities for future lines of research, including systematic reviews and meta-analyses on specific topics, such as biomarkers clinical relevance and secondary prevention approaches.

\section{Conclusions}

Lacunar ischemic strokes are not only a prevalent type of stroke but could also be the first clinical manifestation of SVD, which causes severe physical and cognitive impairment in the long-term. However, not all small subcortical lesions are due to SVD, and improvement in the diagnostic work-up, including advanced imaging, is desirable to exclude other concomitant causes. Translational research is often necessary to develop of new treatments, but optimal animal models of lacunar strokes, mimicking the same underlying mechanisms, are lacking at the moment. On the clinical side, surrogate markers of vascular functions in SVD in neuroimaging are not fully developed and validated. More efforts are needed in the future to encourage the external validation of new SVD markers and include them in large multicenter prospective studies, including RCTs. Using homogenous definitions of SVD markers, standards in the acquisition and processing of SVD imaging are crucial for interpretating and comparing different studies.

Author Contributions: Conceptualization, S.R. and A.A.; methodology, S.R. and A.A.; writing, S.R. and A.R.-V.; review, X.U. and A.A.; supervision, A.A. All authors have read and agreed to the published version of the manuscript.

Funding: This work was partially developed at the building Centro Esther Koplowitz, Barcelona, CERCA Programme/Generalitat de Catalunya.

Institutional Review Board Statement: Not applicable.

Informed Consent Statement: Not applicable.

Conflicts of Interest: The authors declare no conflict of interest.

\section{References}

1. Arboix, A.; Martí-Vilalta, J.L. New Concepts in Lacunar Stroke Etiology: The Constellation of Small-Vessel Arterial Disease. Cerebrovasc. Dis. 2004, 17 (Suppl. 1), 58-62. [CrossRef] [PubMed]

2. Norrving, B. Long-Term Prognosis after Lacunar Infarction. Lancet Neurol. 2003, 2, 238-245. [CrossRef]

3. Blanco-Rojas, L.; Arboix, A.; Canovas, D.; Grau-Olivares, M.; Oliva Morera, J.C.; Parra, O. Cognitive Profile in Patients with a First-Ever Lacunar Infarct with and without Silent Lacunes: A Comparative Study. BMC Neurol. 2013, 13. [CrossRef] [PubMed]

4. Wardlaw, J.M.; Smith, C.; Dichgans, M. Small Vessel Disease: Mechanisms and Clinical Implications. Lancet Neurol. 2019, 18, 684-696. [CrossRef]

5. Wardlaw, J.M.; Smith, E.E.; Biessels, G.J.; Cordonnier, C.; Fazekas, F.; Frayne, R.; Lindley, R.I.; O’Brien, J.T.; Barkhof, F.; Benavente, O.R.; et al. Neuroimaging Standards for Research into Small Vessel Disease and Its Contribution to Ageing and Neurodegeneration. Lancet Neurol. 2013, 12, 822-838. [CrossRef] 
6. Bailey, E.L.; Mcculloch, J.; Sudlow, C.; Wardlaw, J.M. Potential Animal Models of Lacunar Stroke: A Systematic Review. Stroke 2009, 40, e451-e458. [CrossRef]

7. Mustapha, M.; Nassir, C.M.N.C.M.; Aminuddin, N.; Safri, A.A.; Ghazali, M.M. Cerebral Small Vessel Disease (CSVD)—Lessons from the Animal Models. Front. Physiol. 2019, 10, 1317. [CrossRef]

8. Fisher, C.M. Lacunar Strokes and Infarcts: A Review. Neurology 1982, 32, 871-876. [CrossRef]

9. Poirier, J.; Derouesne, C. Cerebral Lacunae. A Proposed New Classification. Clin. Neuropathol. 1984, 3, 266.

10. Lammie, G.A. Hypertensive Cerebral Small Vessel Disease and Stroke. Brain Pathol. 2002, 12, 358-370. [CrossRef]

11. Bailey, E.L.; Smith, C.; Sudlow, C.L.M.; Wardlaw, J.M. Pathology of Lacunar Ischemic Stroke in Humans-A Systematic Review. Brain Pathol. 2012, 22, 583-591. [CrossRef]

12. Adams, H.P., Jr.; Bendixen, B.H.; Kappelle, L.J.; Biller, J.; Love, B.B.; Gordon, D.L.; Marsh, E.E., 3rd. Classification of Subtype of Acute Ischemic Stroke. Definitions for Use in a Multicenter Clinical Trial. TOAST. Trial of Org 10172 in Acute Stroke Treatment. Stroke 1993, 24, 35-41. [CrossRef]

13. Amarenco, P.; Bogousslavsky, J.; Caplan, L.R.; Donnan, G.A.; Wolf, M.E.; Hennerici, M.G. The ASCOD Phenotyping of Ischemic Stroke (Updated ASCO Phenotyping). Cerebrovasc. Dis. 2013, 36, 1-5. [CrossRef]

14. Ay, H.; Furie, K.L.; Singhal, A.; Smith, W.S.; Sorensen, A.G.; Koroshetz, W.J. An Evidence-Based Causative Classification System for Acute Ischemic Stroke. Ann. Neurol. 2005, 58, 688-697. [CrossRef]

15. Humphreys, C.A.; Smith, C.; Wardlaw, J.M. Correlations in Post-Mortem Imaging-Histopathology Studies of Sporadic Human Cerebral Small Vessel Disease: A Systematic Review. Neuropathol. Appl. Neurobiol. 2021. [CrossRef]

16. Wardlaw, J.M.; Smith, C.; Dichgans, M. Mechanisms of Sporadic Cerebral Small Vessel Disease: Insights from Neuroimaging Lancet Neurol. 2013, 12, 483-497. [CrossRef]

17. Regenhardt, R.W.; Das, A.S.; Lo, E.H.; Caplan, L.R. Advances in Understanding the Pathophysiology of Lacunar Stroke: A Review. JAMA Neurol. 2018, 75, 1273-1281. [CrossRef]

18. Caplan, L.R. Lacunar Infarction and Small Vessel Disease: Pathology and Pathophysiology. J. Stroke 2015, 17, 2-6. [CrossRef]

19. Xie, W.; Wang, C.; Liu, S.; Tang, R.; Chai, S.; Guo, Y.; Qian, T.; Chang, B.; Yang, Q.; Fan, Z.; et al. Visualization of Lenticulostriate Artery by Intracranial Dark-Blood Vessel Wall Imaging and Its Relationships with Lacunar Infarction in Basal Ganglia: A Retrospective Study. Eur. Radiol. 2021, 31, 5629-5639. [CrossRef]

20. Ling, C.; Fang, X.; Kong, Q.; Sun, Y.; Wang, B.; Zhuo, Y.; An, J.; Zhang, W.; Wang, Z.; Zhang, Z.; et al. Lenticulostriate Arteries and Basal Ganglia Changes in Cerebral Autosomal Dominant Arteriopathy with Subcortical Infarcts and Leukoencephalopathy, a High-Field Mri Study. Front. Neurol. 2019, 10, 1-9. [CrossRef]

21. Arboix, A.; Altés, E.; García-Eroles, L.; Massons, J. Clinical Study of Lacunar Infarcts in Non-Hypertensive Patients. J. Stroke Cerebrovasc. Dis. 2003, 12, 232-236. [CrossRef] [PubMed]

22. Zonneveld, T.P.; Richard, E.; Vergouwen, M.D.I.; Nederkoorn, P.J.; de Haan, R.; Roos, Y.B.; Kruyt, N.D. Blood Pressure-Lowering Treatment for Preventing Recurrent Stroke, Major Vascular Events, and Dementia in Patients with a History of Stroke or Transient Ischaemic Attack. Cochrane Database Syst. Rev. 2018, 7, CD007858. [CrossRef] [PubMed]

23. Sps, T.; Group, S. Blood-Pressure Targets in Patients with Recent Lacunar Stroke: The SPS3 Randomised Trial. Lancet 2013, 382, 507-515. [CrossRef]

24. Petrone, L.; Nannoni, S.; Del Bene, A.; Palumbo, V.; Inzitari, D. Branch Atheromatous Disease: A Clinically Meaningful, yet Unproven Concept. Cerebrovasc. Dis. 2016, 41, 87-95. [CrossRef] [PubMed]

25. Caplan, L.R. Intracranial Branch Atheromatous Disease: A Neglected, Understudied, and Underused Concept. Neurology 1989, 39, 1246-1250. [CrossRef]

26. Amarenco, P.; Bogousslavsky, J.; Callahan, A.; Goldstein, L.B.; Hennerici, M.; Rudolph, A.E.; Sillesen, H.; Simunovic, L.; Szarek, M.; Welch, K.M.A.; et al. High-Dose Atorvastatin after Stroke or Transient Ischemic Attack. Curr. Atheroscler. Rep. 2007, 9 , 96. [CrossRef]

27. Suzuki, T.; Natori, T.; Sasaki, M.; Miyazawa, H.; Narumi, S.; Ito, K.; Kamada, A.; Yoshida, M.; Tsuda, K.; Yoshioka, K.; et al. Evaluating Recanalization of Relevant Lenticulostriate Arteries in Acute Ischemic Stroke Using High-Resolution MRA at 7T. Int. J. Stroke 2021, 16, 1039-1046. [CrossRef]

28. Rudilosso, S.; Laredo, C.; Mancosu, M.; Moya-Planas, N.; Zhao, Y.; Chirife, O.; Chamorro, Á.; Urra, X. Cerebral Perfusion and Compensatory Blood Supply in Patients with Recent Small Subcortical Infarcts. J. Cereb. Blood Flow Metab. 2019, 39, 1326-1335. [CrossRef]

29. Rydén, L.; Sacuiu, S.; Wetterberg, H.; Najar, J.; Guo, X.; Kern, S.; Zettergren, A.; Shams, S.; Pereira, J.B.; Wahlund, L.-O.; et al. Atrial Fibrillation, Stroke, and Silent Cerebrovascular Disease: A Population-Based MRI Study. Neurology 2021, 97, e1608-e1619. [CrossRef]

30. Lee, J.H.; Kim, Y.J.; Moon, Y.; Cho, H.J.; Kim, H.Y. Acute Simultaneous Multiple Lacunar Infarcts: A Severe Disease Entity in Small Artery Disease. Eur. Neurol. 2012, 67, 303-311. [CrossRef]

31. Stewart, C.R.; Stringer, M.S.; Shi, Y.; Thrippleton, M.J.; Wardlaw, J.M. Associations Between White Matter Hyperintensity Burden, Cerebral Blood Flow and Transit Time in Small Vessel Disease: An Updated Meta-Analysis. Front. Neurol. 2021, 12, 647848. [CrossRef] 
32. Wardlaw, J.; Bath, P.M.W.; Doubal, F.; Heye, A.; Sprigg, N.; Woodhouse, L.J.; Blair, G.; Appleton, J.; Cvoro, V.; England, T.; et al. Protocol: The Lacunar Intervention Trial 2 (LACI-2). A Trial of Two Repurposed Licenced Drugs to Prevent Progression of Cerebral Small Vessel Disease. Eur. Stroke J. 2020, 5. [CrossRef]

33. Roseborough, A.D.; Rasheed, B.; Jung, Y.; Nishimura, K.; Pinsky, W.; Langdon, K.D.; Hammond, R.; Pasternak, S.H.; Khan, A.R.; Whitehead, S.N. Microvessel Stenosis, Enlarged Perivascular Spaces, and Fibrinogen Deposition Are Associated with Ischemic Periventricular White Matter Hyperintensities. Brain Pathol. 2021, 32, e13017. [CrossRef]

34. Simpson, J.E.; Wharton, S.B.; Cooper, J.; Gelsthorpe, C.; Baxter, L.; Forster, G.; Shaw, P.J.; Savva, G.; Matthews, F.E.; Brayne, C.; et al. Alterations of the Blood-Brain Barrier in Cerebral White Matter Lesions in the Ageing Brain. Neurosci. Lett. 2010, 486, $246-251$. [CrossRef]

35. Low, A.; Mak, E.; Rowe, J.B.; Markus, H.S.; O’Brien, J.T. Inflammation and Cerebral Small Vessel Disease: A Systematic Review. Ageing Res. Rev. 2019, 53, 100916. [CrossRef]

36. Wardlaw, J.M. Blood-Brain Barrier and Cerebral Small Vessel Disease. J. Neurol. Sci. 2010, 299, 66-71. [CrossRef]

37. Hainsworth, A.H.; Minett, T.; Andoh, J.; Forster, G.; Bhide, I.; Barrick, T.R.; Elderfield, K.; Jeevahan, J.; Markus, H.S.; Bridges, L.R. Neuropathology of White Matter Lesions, Blood-Brain Barrier Dysfunction, and Dementia. Stroke 2017, 48, 2799-2804. [CrossRef]

38. Wharton, S.B.; Simpson, J.E.; Brayne, C.; Ince, P.G. Age-Associated White Matter Lesions: The MRC Cognitive Function and Ageing Study. Brain Pathol. 2015, 25, 35-43. [CrossRef]

39. Wiseman, S.; Marlborough, F.; Doubal, F.; Webb, D.J.; Wardlaw, J. Blood Markers of Coagulation, Fibrinolysis, Endothelial Dysfunction and Inflammation in Lacunar Stroke versus Non-Lacunar Stroke and Non-Stroke: Systematic Review and MetaAnalysis. Cerebrovasc. Dis. 2014, 37, 64-75. [CrossRef]

40. Kelly, P.; Weimar, C.; Lemmens, R.; Murphy, S.; Purroy, F.; Arsovska, A.; Bornstein, N.M.; Czlonkowska, A.; Fischer, U.; Fonseca, A.C.; et al. Colchicine for Prevention of Vascular Inflammation in Non-CardioEmbolic Stroke (CONVINCE)-Study Protocol for a Randomised Controlled Trial. Eur. Stroke J. 2021, 6, 222-228. [CrossRef]

41. Chamorro, Á.; Amaro, S.; Castellanos, M.; Segura, T.; Arenillas, J.; Martí-Fábregas, J.; Gállego, J.; Krupinski, J.; Gomis, M.; Cánovas, D.; et al. Safety and Efficacy of Uric Acid in Patients with Acute Stroke (URICO-ICTUS): A Randomised, Double-Blind Phase 2b/3 Trial. Lancet Neurol. 2014, 13, 453-460. [CrossRef]

42. Ridker, P.M.; Thuren, T.; Zalewski, A.; Libby, P. Interleukin-1 $\beta$ Inhibition and the Prevention of Recurrent Cardiovascular Events: Rationale and Design of the Canakinumab Anti-Inflammatory Thrombosis Outcomes Study (CANTOS). Am. Heart J. 2011, 162, 597-605. [CrossRef] [PubMed]

43. Rudilosso, S.; Urra, X.; San Roman, L.; Laredo, C.; Lopez-Rueda, A.; Amaro, S.; Oleaga, L.; Chamorro, A. Perfusion Deficits and Mismatch in Patients with Acute Lacunar Infarcts Studied with Whole-Brain CT Perfusion. Am. J. Neuroradiol. 2015, 36, 1407-1412. [CrossRef] [PubMed]

44. Förster, A.; Kerl, H.U.; Wenz, H.; Brockmann, M.A.; Nölte, I.; Groden, C. Diffusion- and Perfusion-Weighted Imaging in Acute Lacunar Infarction: Is There a Mismatch? PLoS ONE 2013, 8, e77428. [CrossRef]

45. Förster, A.; Mürle, B.; Böhme, J.; Al-Zghloul, M.; Kerl, H.U.; Wenz, H.; Groden, C. Perfusion-Weighted Imaging and Dynamic 4D Angiograms for the Estimation of Collateral Blood Flow in Lacunar Infarction. J. Cereb. Blood Flow Metab. 2016, 36, $1744-1754$. [CrossRef]

46. Huang, Y.C.; Lee, J.D.; Pan, Y.T.; Weng, H.H.; Yang, J.T.; Lin, L.C.; Tsai, Y.H. Perfusion Defects and Collateral Flow Patterns in Acute Small Subcortical Infarction: A 4D Dynamic MRI Study. Transl. Stroke Res. 2021. [CrossRef]

47. Smirnov, M.; Destrieux, C.; Maldonado, I.L. Cerebral White Matter Vasculature: Still Uncharted? Brain 2021, 144, 3561-3575. [CrossRef]

48. Jouvent, E.; Duering, M.; Chabriat, H. Cerebral Autosomal Dominant Arteriopathy With Subcortical Infarcts and Leukoencephalopathy: Lessons from Neuroimaging. Stroke 2020, 51, 21-28. [CrossRef]

49. Choi, J.C. Genetics of Cerebral Small Vessel Disease. J. Stroke 2015, 17, 7-16. [CrossRef]

50. Muiño, E.; Fernández-Cadenas, I.; Arboix, A. Contribution of "Omic" Studies to the Understanding of CADASIL. A Systematic Review. Int. J. Mol. Sci. 2021, 22, 7357. [CrossRef]

51. Mancuso, M.; Arnold, M.; Bersano, A.; Burlina, A.; Chabriat, H.; Debette, S.; Enzinger, C.; Federico, A.; Filla, A.; Finsterer, J.; et al. Monogenic Cerebral Small-Vessel Diseases: Diagnosis and Therapy. Consensus Recommendations of the European Academy of Neurology. Eur. J. Neurol. 2020, 27, 909-927. [CrossRef]

52. Smith, E.E.; Biessels, G.J.; De Guio, F.; de Leeuw, F.E.; Duchesne, S.; Düring, M.; Frayne, R.; Ikram, M.A.; Jouvent, E.; MacIntosh, B.J.; et al. Harmonizing Brain Magnetic Resonance Imaging Methods for Vascular Contributions to Neurodegeneration. Alzheimer's Dement. Diagn. Assess. Dis. Monit. 2019, 11, 191-204. [CrossRef]

53. Lee, J.M.; Grabb, M.C.; Zipfel, G.J.; Choi, D.W. Brain Tissue Responses to Ischemia. J. Clin. Investig. 2000, 106, 723-731. [CrossRef]

54. Kim, J.G.; Choi, H.; Sohn, S.Y.; Kim, D.H.; Lee, S.J. Transient Ischemic Attacks Preceding Acute Lacunar Infarction. Eur. Neurol. 2016, 76, 278-283. [CrossRef]

55. Rudilosso, S.; Urra, X.; Chirife, O.; Chamorro, Á. Altered Brain Computed Tomography Perfusion in Patients with Fluctuating Lacunar Syndrome and Normal Magnetic Resonance Imaging. JAMA Neurol. 2016, 73, 348-349. [CrossRef]

56. Donnan, G.A.; O'Malley, H.M.; Quang, L.; Hurley, S.; Bladin, P.F. The Capsular Warning Syndrome: Pathogenesis and Clinical Features. Neurology 1993, 43, 957-962. [CrossRef] 
57. Camps-Renom, P.; Delgado-Mederos, R.; Martínez-Domeño, A.; Prats-Sánchez, L.; Cortés-Vicente, E.; Simón-Talero, M.; Arboix, A.; Ois, Á.; Purroy, F.; Martí-Fàbregas, J. Clinical Characteristics and Outcome of the Capsular Warning Syndrome: A Multicenter Study. Int. J. Stroke 2015, 10, 571-575. [CrossRef]

58. Saposnik, G.; De Tilly, L.N.; Caplan, L.R. Pontine Warning Syndrome. Arch. Neurol. 2008, 65, 1375-1377. [CrossRef]

59. Muengtaweepongsa, S.; Singh, N.N.; Cruz-Flores, S. Pontine Warning Syndrome: Case Series and Review of Literature. J. Stroke Cerebrovasc. Dis. 2010, 19, 353-356. [CrossRef]

60. He, L.; Xu, R.; Wang, J.; Zhang, L.; Zhang, L.; Zhou, F.; Dong, W. Capsular Warning Syndrome: Clinical Analysis and Treatment BMC Neurol. 2019, 19, 1-7. [CrossRef]

61. Rudilosso, S.; Olivera, M.; Esteller, D.; Laredo, C.; Amaro, S.; Llull, L.; Renú, A.; Obach, V.; Vera, V.; Rodríguez, A.; et al. Susceptibility Vessel Sign in Deep Perforating Arteries in Patients with Recent Small Subcortical Infarcts. J. Stroke Cerebrovasc. Dis. 2021, 30, 1-7. [CrossRef]

62. Jiang, S.; Yan, Y.; Yang, T.; Zhu, Q.; Wang, C.; Bai, X.; Hao, Z.; Zhang, S.; Yang, Q.; Fan, Z.; et al. Plaque Distribution Correlates with Morphology of Lenticulostriate Arteries in Single Subcortical Infarctions. Stroke 2020, 51, 2801-2809. [CrossRef]

63. Zhang, Z.; Fan, Z.; Kong, Q.; Xiao, J.; Wu, F.; An, J.; Yang, Q.; Li, D.; Zhuo, Y. Visualization of the Lenticulostriate Arteries at 3T Using Black-Blood T1-Weighted Intracranial Vessel Wall Imaging: Comparison with 7T TOF-MRA. Eur. Radiol. 2019, 29, 1452-1459. [CrossRef]

64. Wardlaw, J.M.; Doubal, F.; Armitage, P.; Chappell, F.; Carpenter, T.; Muñoz Maniega, S.; Farrall, A.; Sudlow, C.; Dennis, M.; Dhillon, B. Lacunar Stroke Is Associated with Diffuse Blood-Brain Barrier Dysfunction. Ann. Neurol. 2009, 65, 194-202. [CrossRef]

65. Blair, G.W.; Thrippleton, M.J.; Shi, Y.; Hamilton, I.; Stringer, M.; Chappell, F.; Dickie, D.A.; Andrews, P.; Marshall, I.; Doubal, F.N.; et al. Intracranial Hemodynamic Relationships in Patients with Cerebral Small Vessel Disease. Neurology 2020, 94 , e2258-e2269. [CrossRef] [PubMed]

66. Stevenson, S.F.; Doubal, F.N.; Shuler, K.; Wardlaw, J.M. A Systematic Review of Dynamic Cerebral and Peripheral Endothelial Function in Lacunar Stroke versus Controls. Stroke 2010, 41, 434-442. [CrossRef]

67. Moreau, F.; Patel, S.; Lauzon, M.L.; McCreary, C.R.; Goyal, M.; Frayne, R.; Demchuk, A.M.; Coutts, S.B.; Smith, E.E. Cavitation after Acute Symptomatic Lacunar Stroke Depends on Time, Location, and MRI Sequence. Stroke 2012, 43, 1837-1842. [CrossRef]

68. Lee, K.J.; Jung, H.; Oh, Y.S.; Lim, E.Y.; Cho, A.H. The Fate of Acute Lacunar Lesions in Terms of Shape and Size. J. Stroke Cerebrovasc. Dis. 2017, 26, 1254-1257. [CrossRef]

69. Gattringer, T.; Valdes Hernandez, M.; Heye, A.; Armitage, P.A.; Makin, S.; Chappell, F.; Pinter, D.; Doubal, F.; Enzinger, C.; Fazekas, F.; et al. Predictors of Lesion Cavitation After Recent Small Subcortical Stroke. Transl. Stroke Res. 2020, 11, 402-411. [CrossRef]

70. Benjamin, P.; Trippier, S.; Lawrence, A.J.; Lambert, C.; Zeestraten, E.; Williams, O.A.; Patel, B.; Morris, R.G.; Barrick, T.R.; MacKinnon, A.D.; et al. Lacunar Infarcts, but Not Perivascular Spaces, Are Predictors of Cognitive Decline in Cerebral SmallVessel Disease. Stroke 2018, 49, 586-593. [CrossRef]

71. del C. Valdés Hernández, M.; Maconick, L.C.; Muñoz Maniega, S.; Wang, X.; Wiseman, S.; Armitage, P.A.; Doubal, F.N.; Makin, S.; Sudlow, C.L.; Dennis, M.S.; et al. A Comparison of Location of Acute Symptomatic vs. "silent" Small Vessel Lesions. Int. J. Stroke 2015, 10, 1044-1050. [CrossRef] [PubMed]

72. Duering, M.; Csanadi, E.; Gesierich, B.; Jouvent, E.; Hervé, D.; Seiler, S.; Belaroussi, B.; Ropele, S.; Schmidt, R.; Chabriat, H.; et al. Incident Lacunes Preferentially Localize to the Edge of White Matter Hyperintensities: Insights into the Pathophysiology of Cerebral Small Vessel Disease. Brain 2013, 136, 2717-2726. [CrossRef]

73. Keith, J.; Gao, F.Q.; Noor, R.; Kiss, A.; Balasubramaniam, G.; Au, K.; Rogaeva, E.; Masellis, M.; Black, S.E. Collagenosis of the Deep Medullary Veins: An Underrecognized Pathologic Correlate of White Matter Hyperintensities and Periventricular Infarction? J. Neuropathol. Exp. Neurol. 2017, 76, 299-312. [CrossRef] [PubMed]

74. Tuttolomondo, A.; Di Sciacca, R.; Di Raimondo, D.; Renda, C.; Pinto, A.; Licata, G. Inflammation as a Therapeutic Target in Acute Ischemic Stroke Treatment. Curr. Top. Med. Chem. 2009, 9, 1240-1260. [CrossRef]

75. Shoamanesh, A.; Preis, S.R.; Beiser, A.S.; Kase, C.S.; Wolf, P.A.; Vasan, R.S.; Benjamin, E.J.; Seshadri, S.; Romero, J.R. Circulating Biomarkers and Incident Ischemic Stroke in the Framingham Offspring Study. Neurology 2016, 87, 1206-1211. [CrossRef]

76. Mengozzi, M.; Kirkham, F.A.; Girdwood, E.E.R.; Bunting, E.; Drazich, E.; Timeyin, J.; Ghezzi, P.; Rajkumar, C. C-Reactive Protein Predicts Further Ischemic Events in Patients With Transient Ischemic Attack or Lacunar Stroke. Front. Immunol. 2020, 11, 1-7. [CrossRef]

77. Elkind, M.S.V.; Luna, J.M.; McClure, L.A.; Zhang, Y.; Coffey, C.S.; Roldan, A.; Del Brutto, O.H.; Pretell, E.J.; Pettigrew, L.C.; Meyer, B.C.; et al. C-Reactive Protein as a Prognostic Marker after Lacunar Stroke Levels of Inflammatory Markers in the Treatment of Stroke Study. Stroke 2014, 45, 707-716. [CrossRef]

78. Boehme, A.K.; McClure, L.A.; Zhang, Y.; Luna, J.M.; Del Brutto, O.H.; Benavente, O.R.; Elkind, M.S.V. Inflammatory Markers and Outcomes after Lacunar Stroke: Levels of Inflammatory Markers in Treatment of Stroke Study. Stroke 2016, 47, 659-667. [CrossRef]

79. Lindgren, A.; Lindoff, C.; Norrving, B.; Åstedt, B.; Johansson, B.B. Tissue Plasminogen Activator and Plasminogen Activator Inhibitor-1 in Stroke Patients. Stroke 1996, 27, 1066-1071. [CrossRef] 
80. Salobir, B.; Šabovič, M.; Peternel, P.; Stegnar, M. Vascular Bed Specific Alterations in Coagulation and Fibrinolytic Parameters in Young Women Following Myocardial Infarction, Lacunar Cerebral Infarction and Deep Vein Thrombosis. Pathophysiol. Haemost. Thromb. 2003, 33, 96-101. [CrossRef]

81. Jood, K.; Ladenvall, P.; Tjärnlund-Wolf, A.; Ladenvall, C.; Andersson, M.; Nilsson, S.; Blomstrand, C.; Jern, C. Fibrinolytic Gene Polymorphism and Ischemic Stroke. Stroke 2005, 36, 2077-2081. [CrossRef] [PubMed]

82. Yokokawa, H.; Goto, A.; Terui, K.; Funami, Y.; Watanabe, K.; Yasumura, S. Prevalence of Metabolic Syndrome and Serum Marker Levels in Patients with Four Subtypes of Cerebral Infarction in Japan. J. Clin. Neurosci. 2008, 15, 769-773. [CrossRef]

83. Ilhan, D.; Özbabalik, D.; Gulcan, E.; Özdemir, Ö.; Gülbaçs, Z. Evaluation of Platelet Activation, Coagulation, and Fibrinolytic Activation in Patients with Symptomatic Lacunar Stroke. Neurologist 2010, 16, 188-191. [CrossRef]

84. Kilpatrick, T.J.; Matkovic, Z.; Davis, S.M.; McGrath, C.M.; Dauer, R.J. Hematologic Abnormalities Occur in Both Cortical and Lacunar Infarction. Stroke 1993, 24, 1945-1950. [CrossRef]

85. Beamer, N.B.; Coull, B.M.; Clark, W.M.; Hazel, J.S.; Silberger, J.R. Interleukin-6 and Interleukin-1 Receptor Antagonist in Acute Stroke. Ann. Neurol. 1995, 37, 800-805. [CrossRef]

86. Bath, P.M.W.; Blann, A.; Smith, N.; Butterworth, R.J. Von Willebrand Factor, P-Selectin and Fibrinogen Levels in Patients with Acute Ischaemic and Haemorrhagic Stroke, and Their Relationship with Stroke Sub-Type and Functional Outcome. Platelets 1998, 9, 155-159. [CrossRef]

87. Kataoka, S.; Hirose, G.; Hori, A.; Shirakawa, T.; Saigan, T. Activation of Thrombosis and Fibrinolysis Following Brain Infarction. J. Neurol. Sci. 2000, 181, 82-88. [CrossRef]

88. Alvarez-Perez, F.J.; Castelo-Branco, M.; Alvarez-Sabin, J. Usefulness of Measurement of Fibrinogen, D-Dimer, D-Dimer/Fibrinogen Ratio, C Reactive Protein and Erythrocyte Sedimentation Rate to Assess the Pathophysiology and Mechanism of Ischaemic Stroke. J. Neurol. Neurosurg. Psychiatry 2011, 82, 986-992. [CrossRef]

89. Beer, C.; Blacker, D.; Hankey, G.J.; Puddey, I.B. Association of Clinical and Aetiologic Subtype of Acute Ischaemic Stroke with Inflammation, Oxidative Stress and Vascular Function: A Cross-Sectional Observational Study. Med. Sci. Monit. 2011, 17, CR467. [CrossRef]

90. Zhang, B.; Zhang, W.Z.; Li, X.; Pu, S.X.; Yin, J.R.; Yang, N.; Yi, Y.H.; Gao, Q.C.; Gao, C. Admission Markers Predict Lacunar and Non-Lacunar Stroke in Young Patients. Thromb. Res. 2011, 128, 14-17. [CrossRef]

91. Datta, A.; Chen, C.P.; Sze, S.K. Discovery of Prognostic Biomarker Candidates of Lacunar Infarction by Quantitative Proteomics of Microvesicles Enriched Plasma. PLoS ONE 2014, 9, e94663. [CrossRef] [PubMed]

92. Takano, K.; Yamaguchi, T.; Uchida, K. Markers of a Hypercoagulable State Following Acute Ischemic Stroke. Stroke 1992, 23, 194-198. [CrossRef] [PubMed]

93. Ageno, W.; Finazzi, S.; Steidl, L.; Biotti, M.G.; Mera, V.; D’Eril, G.V.M.; Venco, A. Plasma Measurement of D-Dimer Levels for the Early Diagnosis of Ischemic Stroke Subtypes. Arch. Intern. Med. 2002, 162, 2589-2593. [CrossRef]

94. Montaner, J.; Perea-Gainza, M.; Delgado, P.; Ribó, M.; Chacón, P.; Rosell, A.; Quintana, M.; Palacios, M.E.; Molina, C.A.; Alvarez-Sabín, J. Etiologic Diagnosis of Ischemic Stroke Subtypes with Plasma Biomarkers. Stroke 2008, 39, 2280-2287. [CrossRef]

95. Brouns, R.; Van Den Bossche, J.; De Surgeloose, D.; Sheorajpanday, R.; De Deyn, P.P. Clinical and Biochemical Diagnosis of Small-Vessel Disease in Acute Ischemic Stroke. J. Neurol. Sci. 2009, 285, 185-190. [CrossRef]

96. Isenegger, J.; Meier, N.; Lämmle, B.; Alberio, L.; Fischer, U.; Nedeltchev, K.; Gralla, J.; Kohler, H.P.; Mattle, H.P.; Arnold, M. D-Dimers Predict Stroke Subtype When Assessed Early. Cerebrovasc. Dis. 2009, 29, 82-86. [CrossRef]

97. Eikelboom, J.W.; Hankey, G.J.; Anand, S.S.; Lofthouse, E.; Staples, N.; Baker, R.I. Association between High Homocyst(e)Ine and Ischemic Stroke Due to Large- and Small-Artery Disease but Not Other Etiologic Subtypes of Ischemic Stroke. Stroke 2000, 31, 1069-1075. [CrossRef]

98. Hassan, A.; Gormley, K.; O’Sullivan, M.; Knight, J.; Sham, P.; Vallance, P.; Bamford, J.; Markus, H. Endothelial Nitric Oxide Gene Haplotypes and Risk of Cerebral Small-Vessel Disease. Stroke 2004, 35, 654-659. [CrossRef]

99. Parnetti, L.; Caso, V.; Santucci, A.; Corea, F.; Lanari, A.; Floridi, A.; Conte, C.; Bottiglieri, T. Mild Hyperhomocysteinemia Is a Risk-Factor in All Etiological Subtypes of Stroke. Neurol. Sci. 2004, 25, 13-17. [CrossRef]

100. Khan, U.; Hassan, A.; Vallance, P.; Markus, H.S. Asymmetric Dimethylarginine in Cerebral Small Vessel Disease. Stroke 2007, 38, 411-413. [CrossRef]

101. Khan, U.; Crossley, C.; Kalra, L.; Rudd, A.; Wolfe, C.D.A.; Collinson, P.; Markus, H.S. Homocysteine and Its Relationship to Stroke Subtypes in a UK Black Population: The South London Ethnicity and Stroke Study. Stroke 2008, 39, 2943-2949. [CrossRef]

102. Jeong, S.K.; Kim, D.H.; Cho, Y.I. Homocysteine and Pulsatility Index in Lacunar Infarction. Clin. Neurol. Neurosurg. 2011, 113, 459-463. [CrossRef]

103. Pavlovic, A.M.; Pekmezovic, T.; Obrenovic, R.; Novakovic, I.; Tomic, G.; Mijajlovic, M.; Sternic, N. Increased Total Homocysteine Level Is Associated with Clinical Status and Severity of White Matter Changes in Symptomatic Patients with Subcortical Small Vessel Disease. Clin. Neurol. Neurosurg. 2011, 113, 711-715. [CrossRef]

104. Lavallée, P.C.; Labreuche, J.; Faille, D.; Huisse, M.G.; Nicaise-Roland, P.; Dehoux, M.; Gongora-Rivera, F.; Jaramillo, A.; Brenner, D.; Deplanque, D.; et al. Circulating Markers of Endothelial Dysfunction and Platelet Activation in Patients with Severe Symptomatic Cerebral Small Vessel Disease on Behalf of the Lacunar-B.I.C.H.A.T. Investigators. Cerebrovasc. Dis. 2013, 36, 131-138. [CrossRef]

105. Hanson, E.; Jood, K.; Karlsson, S.; Nilsson, S.; Blomstrand, C.; Jern, C. Plasma Levels of von Willebrand Factor in the Etiologic Subtypes of Ischemic Stroke. J. Thromb. Haemost. 2011, 9, 275-281. [CrossRef] 
106. Kozuka, K.; Kohriyama, T.; Ikeda, J.; Nakamura, S.; Nomura, E.; Kajikawa, H. Endothelial Markers and Adhesion Molecules in Acute Ischemic Stroke-Sequential Change and Differences in Stroke Subtype. Atherosclerosis 2002, 161, 161-168. [CrossRef]

107. Tsai, N.W.; Chang, W.N.; Shaw, C.F.; Jan, C.R.; Huang, C.R.; Chen, S.D.; Chuang, Y.C.; Lee, L.H.; Lu, C.H. The Value of Leukocyte Adhesion Molecules in Patients after Ischemic Stroke. J. Neurol. 2009, 256, 1296-1302. [CrossRef]

108. Turgut, B.; Turgut, N.; Çelik, Y.; Tekgündüz, E.; Pamuk, G.E.; Demir, M. Differences in Platelet-Leukocyte Aggregates among Subtypes of Acute Cerebral Ischemia. J. Neurol. Sci. 2011, 305, 126-130. [CrossRef]

109. Castellanos, M.; Castillo, J.; García, M.M.; Leira, R.; Serena, J.; Chamorro, A.; Dávalos, A. Inflammation-Mediated Damage in Progressing Lacunar Infarctions a Potential Therapeutic Target. Stroke 2002, 33, 982-987. [CrossRef]

110. Supanc, V.; Biloglav, Z.; Kes, V.; Demarin, V. Role of Cell Adhesion Molecules in Acute Ischemic Stroke. Ann. Saudi Med. 2011, 31, 365-370. [CrossRef]

111. Rouhl, R.P.W.; Damoiseaux, J.G.M.C.; Lodder, J.; Theunissen, R.O.M.F.I.H.; Knottnerus, I.L.H.; Staals, J.; Henskens, L.H.G.; Kroon, A.A.; de Leeuw, P.W.; Tervaert, J.W.C.; et al. Vascular Inflammation in Cerebral Small Vessel Disease. Neurobiol. Aging 2012, 33, 1800-1806. [CrossRef] [PubMed]

112. Brown, C.M.; Bushnell, C.D.; Samsa, G.P.; Goldstein, L.B.; Colton, C.A. Chronic Systemic Immune Dysfunction in AfricanAmericans with Small Vessel-Type Ischemic Stroke. Transl. Stroke Res. 2015, 6, 430-436. [CrossRef] [PubMed]

113. Nakase, T.; Yamazaki, T.; Ogura, N.; Suzuki, A.; Nagata, K. The Impact of Inflammation on the Pathogenesis and Prognosis of Ischemic Stroke. J. Neurol. Sci. 2008, 271, 104-109. [CrossRef] [PubMed]

114. Mitaki, S.; Nagai, A.; Oguro, H.; Yamaguchi, S. C-Reactive Protein Levels Are Associated with Cerebral Small Vessel-Related Lesions. Acta Neurol. Scand. 2016, 133, 68-74. [CrossRef]

115. Intiso, D.; Zarrelli, M.M.; Lagioia, G.; Di Rienzo, F.; De Ambrosio, C.C.; Simone, P.; Tonali, P.; Cioffi, R.P. Tumor Necrosis Factor Alpha Serum Levels and Inflammatory Response in Acute Ischemic Stroke Patients. Neurol. Sci. 2004, 24, 390-396. [CrossRef]

116. Vila, N.; Castillo, J.; Dávalos, A.; Chamorro, Á. Proinflammatory Cytokines and Early Neurological Worsening in Ischemic Stroke. Stroke 2000, 31, 2325-2329. [CrossRef]

117. Salobir, B.; Šabovič, M. Possible Vascular-Bed-Specific Role of Interleukin-6 in Young Women with a History of Myocardial Infarction, Lacunar Cerebral Infarction and Deep Vein Thrombosis. Cytokine 2004, 25, 265-272. [CrossRef]

118. Guldiken, B.; Guldiken, S.; Turgut, B.; Turgut, N.; Demir, M.; Celik, Y.; Arikan, E.; Tugrul, A. The Roles of Oxidized Low-Density Lipoprotein and Interleukin-6 Levels in Acute Atherothrombotic and Lacunar Ischemic Stroke. Angiology 2008, 59, 224-229. [CrossRef]

119. Horie, N.; Maag, A.L.; Hamilton, S.A.; Shichinohe, H.; Bliss, T.M.; Steinberg, G.K. Mouse Model of Focal Cerebral Ischemia Using Endothelin-1. J. Neurosci. Methods 2008, 173, 286-290. [CrossRef]

120. Capone, C.; Faraco, G.; Park, L.; Cao, X.; Davisson, R.L.; Iadecola, C. The Cerebrovascular Dysfunction Induced by Slow Pressor Doses of Angiotensin II Precedes the Development of Hypertension. Am. J. Physiol.-Hear. Circ. Physiol. 2011, 300, H397-H407. [CrossRef]

121. Cipolla, M.J.; Sweet, J.G.; Gokina, N.I.; White, S.L.; Nelson, M.T. Mechanisms of Enhanced Basal Tone of Brain Parenchymal Arterioles during Early Postischemic Reperfusion: Role of ET-1-Induced Peroxynitrite Generation. J. Cereb. Blood Flow Metab. 2013, 33, 1486-1492. [CrossRef]

122. Cui, X.; Chopp, M.; Zacharek, A.; Ning, R.; Ding, X.; Roberts, C.; Chen, J. Endothelial Nitric Oxide Synthase Regulates White Matter Changes via the BDNF/TrKB Pathway after Stroke in Mice. PLoS ONE 2013, 8, e80358. [CrossRef]

123. Rapp, J.H.; Pan, X.M.; Yu, B.; Swanson, R.A.; Higashida, R.T.; Simpson, P.; Saloner, D. Cerebral Ischemia and Infarction from Atheroemboli $<100 \mathrm{Mm}$ in Size. Stroke 2003, 34, 1976-1980. [CrossRef]

124. Wang, C.B.; Hu, H.S.; Liu, Y.M. Animal Study in Combining Targeted-Microbubbles with Low-Frequency Ultrasound for the Treatment of Ischemic Stroke. J. Sichuan Univ. Medical Sci. Ed. 2012, 43, 864-867.

125. Silasi, G.; She, J.; Boyd, J.D.; Xue, S.; Murphy, T.H. A Mouse Model of Small-Vessel Disease That Produces Brain-Wide-Identified Microocclusions and Regionally Selective Neuronal Injury. J. Cereb. Blood Flow Metab. 2015, 35, 734-738. [CrossRef]

126. Walz, W.; Cayabyab, F.S. Neutrophil Infiltration and Matrix Metalloproteinase-9 in Lacunar Infarction. Neurochem. Res. 2017, 42, 2560-2565. [CrossRef]

127. Wen, Z.; Jiang, Y.; Zhang, L.; Xu, X.; Zhao, N.; Xu, X.; Wang, F.; Gao, J.; Yang, G.Y.; Liu, X. The Effect of Anterior Communicating Artery Flow on Neurovascular Injury and Neurobehavioral Outcomes in Mice with Recurrent Stroke. Brain Res. 2019, 1724 [CrossRef]

128. Choi, J.I.; Ha, S.K.; Lim, D.J.; Kim, S.D.; Kim, S.H. S100ß, Matrix Metalloproteinase-9, D-Dimer, and Heat Shock Protein 70 Are Serologic Biomarkers of Acute Cerebral Infarction in a Mouse Model of Transient MCA Occlusion. J. Korean Neurosurg. Soc. 2018, 61, 548-558. [CrossRef]

129. Kwon, S.K.; Ahn, M.; Song, H.J.; Kang, S.K.; Jung, S.B.; Harsha, N.; Jee, S.; Moon, J.Y.; Suh, K.S.; Lee, S.D.; et al. Nafamostat Mesilate Attenuates Transient Focal Ischemia/Reperfusion-Induced Brain Injury via the Inhibition of Endoplasmic Reticulum Stress. Brain Res. 2015, 1627, 12-20. [CrossRef]

130. Hainsworth, A.H.; Markus, H.S. Do in Vivo Experimental Models Reflect Human Cerebral Small Vessel Disease? A Systematic Review. J. Cereb. Blood Flow Metab. 2008, 28, 1877-1891. [CrossRef] 
131. Mencl, S.; Garz, C.; Niklass, S.; Braun, H.; Göb, E.; Homola, G.; Heinze, H.J.; Reymann, K.G.; Kleinschnitz, C.; Schreiber, S. Early Microvascular Dysfunction in Cerebral Small Vessel Disease Is Not Detectable on 3.0 Tesla Magnetic Resonance Imaging: A Longitudinal Study in Spontaneously Hypertensive Stroke-Prone Rats. Exp. Transl. Stroke Med. 2013, 5, 8. [CrossRef]

132. Ayata, C. CADASIL: Experimental Insights from Animal Models. Stroke 2010, 41, S129-S134. [CrossRef]

133. Joutel, A. Pathogenesis of CADASIL: Transgenic and Knock-out Mice to Probe Function and Dysfunction of the Mutated Gene, Notch3, in the Cerebrovasculature. BioEssays 2011, 33, 73-80. [CrossRef]

134. Ruchoux, M.M.; Domenga, V.; Brulin, P.; Maciazek, J.; Limol, S.; Tournier-Lasserve, E.; Joutel, A. Transgenic Mice Expressing Mutant Notch3 Develop Vascular Alterations Characteristic of Cerebral Autosomal Dominant Arteriopathy with Subcortical Infarcts and Leukoencephalopathy. Am. J. Pathol. 2003, 162, 329-342. [CrossRef]

135. Cipolla, L.; Consonni, A.; Guidetti, G.; Canobbio, I.; Okigaki, M.; Falasca, M.; Ciraolo, E.; Hirsch, E.; Balduini, C.; Torti, M. The Proline-Rich Tyrosine Kinase Pyk2 Regulates Platelet Integrin AIlbß3 Outside-in Signaling. J. Thromb. Haemost. 2013, 11, 345-356. [CrossRef]

136. Fisher, C.M. Lacunes: Small, Deep Cerebral Infarcts. Neurology 1965, 15, 774-784. [CrossRef]

137. Fisher, C.M. Pure Sensory Stroke Involving Face, Arm, and Leg. Neurology 1965, 15, 78-80. [CrossRef]

138. Mohr, J.P.; Kase, C.S.; Meckler, R.J.; Fisher, C.M. Sensorimotor Stroke Due to Thalamocapsular Ischemia. Arch. Neurol. 1977, 34, 739-741. [CrossRef]

139. Chamorro, A.; Sacco, R.L.; Mohr, J.P.; Foulkes, M.A.; Kase, C.S.; Tatemichi, T.K.; Wolf, P.A.; Price, T.R.; Hier, D.B. ClinicalComputed Tomographic Correlations of Lacunar Infarction in the Stroke Data Bank. Stroke 1991, 22, 175-181. [CrossRef]

140. Arboix, A.; García-Plata, C.; García-Eroles, L.; Massons, J.; Comes, E.; Oliveres, M.; Targa, C. Clinical Study of 99 Patients with Pure Sensory Stroke. J. Neurol. 2005, 252, 156-162. [CrossRef]

141. Arboix, A.; Bell, Y.; García-Eroles, L.; Massons, J.; Comes, E.; Balcells, M.; Targa, C. Clinical Study of 35 Patients with DysarthriaClumsy Hand Syndrome. J. Neurol. Neurosurg. Psychiatry 2004, 75, 231-234. [CrossRef] [PubMed]

142. Huang, C.Y.; Lui, F.S. Ataxic-Hemiparesis, Localization and Clinical Features. Stroke 1984, 15, 363-366. [CrossRef] [PubMed]

143. Arboix, A. Hemiparesia Atáxica: Estudio de 23 Pacientes. Med. Clin. (Barc) 2004, 122, 342-344. [CrossRef]

144. Fisher, C.M. Ataxic Hemiparesis: A Pathologic Study. Arch. Neurol. 1978, 35, 126-128. [CrossRef] [PubMed]

145. Arboix, A.; Martí-Vilaita, J.L. Lacunar Stroke. Expert Rev. Neurother. 2009, 9, 179-196. [CrossRef] [PubMed]

146. Arboix, A.; López-Grau, M.; Casasnovas, C.; García-Eroles, L.; Massons, J.; Balcells, M. Clinical Study of 39 Patients with Atypical Lacunar Syndrome. J. Neurol. Neurosurg. Psychiatry 2006, 77, 381-384. [CrossRef]

147. Potter, G.; Doubal, F.; Jackson, C.; Sudlow, C.; Dennis, M.; Wardlaw, J. Associations of Clinical Stroke Misclassification ('ClinicalImaging Dissociation') in Acute Ischemic Stroke. Cerebrovasc. Dis. 2010, 29, 395-402. [CrossRef]

148. Ay, H.; Oliveira-Filho, J.; Buonanno, F.S.; Ezzeddine, M.; Schaefer, P.W.; Rordorf, G.; Schwamm, L.H.; Gonzalez, R.G.; Koroshetz, W.J. Diffusion-Weighted Imaging Identifies a Subset of Lacunar Infarction Associated with Embolic Source. Stroke 1999, 30, 2644-2650. [CrossRef]

149. Hofmeister, C.; Hartmann, A.; Marx, P.; Mast, H.; Stapf, C. Predictive Value of Clinical Syndromes for Lacunar Infarcts on Magnetic Resonance Brain Imaging. Acta Neurol. Scand. 2000, 101, 13-18. [CrossRef]

150. Lindgren, A.; Staaf, G.; Geijer, B.; Brockstedt, S.; Ståhlberg, F.; Holtås, S.; Norrving, B. Clinical Lacunar Syndromes as Predictors of Lacunar Infarcts: A Comparison of Acute Clinical Lacunar Syndromes and Findings on Diffusion- Weighted MRI. Acta Neurol. Scand. 2000, 101, 128-134. [CrossRef]

151. Seifert, T.; Enzinger, C.; Storch, M.K.; Pichler, G.; Niederkorn, K.; Fazekas, F. Acute Small Subcortical Infarctions on Diffusion Weighted MRI: Clinical Presentation and Aetiology. J. Neurol. Neurosurg. Psychiatry 2005, 76, 1520-1524. [CrossRef]

152. Wessels, T.; Röttger, C.; Jauss, M.; Kaps, M.; Traupe, H.; Stol, E. Identification of Embolic Stroke Patterns by Diffusion-Weighted MRI in Clinically Defined Lacunar Stroke Syndromes. Stroke 2005, 36, 757-761. [CrossRef]

153. Arboix, A.; Massons, J.; García-Eroles, L.; Targa, C.; Comes, E.; Parra, O. Clinical Predictors of Lacunar Syndrome Not Due to Lacunar Infarction. BMC Neurol. 2010, 10, 31. [CrossRef]

154. Altmann, M.; Thommessen, B.; Rønning, O.M.; Reichenbach, A.S.; Fure, B. Diagnostic Accuracy and Risk Factors of the Different Lacunar Syndromes. J. Stroke Cerebrovasc. Dis. 2014, 23, 2085-2090. [CrossRef]

155. Giacomozzi, S.; Caso, V.; Agnelli, G.; Acciarresi, M.; Alberti, A.; Venti, M.; Mosconi, M.G.; Paciaroni, M. Lacunar Stroke Syndromes as Predictors of Lacunar and Non-Lacunar Infarcts on Neuroimaging: A Hospital-Based Study. Intern. Emerg. Med. 2020, 15, 429-436. [CrossRef]

156. Arba, F.; Mair, G.; Phillips, S.; Sandercock, P.; Wardlaw, J.M. Improving Clinical Detection of Acute Lacunar Stroke: Analysis from the IST-3. Stroke 2020, 51, 1411-1418. [CrossRef]

157. Nelson, R.F.; Pullicino, P.; Kendall, B.E.; Marshall, J. Computed Tomography in Patients Presenting with Lacunar Syndromes. Stroke 1980, 11, 256-261. [CrossRef]

158. Rothrock, J.F.; Lyden, P.D.; Hesselink, J.R.; Brown, J.J.; Healy, M.E. Brain Magnetic Resonance Imaging in the Evaluation of Lacunar Stroke. Stroke 1987, 18, 781-786. [CrossRef]

159. Noguchi, K.; Nagayoshi, T.; Watanabe, N.; Kanazawa, T.; Toyoshima, S.; Morijiri, M.; Shojaku, H.; Shimizu, M.; Seto, H. Diffusion-Weighted Echo-Planar MRI of Lacunar Infarcts. Neuroradiology 1998, 40, 448-451. [CrossRef]

160. Schonewille, W.J.; Tuhrim, S.; Singer, M.B.; Atlas, S.W. Diffusion-Weighted MRI in Acute Lacunar Syndromes: A ClinicalRadiological Correlation Study. Stroke 1999, 30, 2066-2069. [CrossRef] 
161. Tan, M.Y.Q.; Singhal, S.; Ma, H.; Chandra, R.V.; Cheong, J.; Clissold, B.B.; Ly, J.; Srikanth, V.; Phan, T.G. Examining Subcortical Infarcts in the Era of Acute Multimodality CT Imaging. Front. Neurol. 2016, 7, 220. [CrossRef]

162. Arsava, E.M.; Ballabio, E.; Benner, T.; Cole, J.W.; Delgado-Martinez, M.P.; Dichgans, M.; Fazekas, F.; Furie, K.L.; Illoh, K.; Jood, K.; et al. The Causative Classification of Stroke System: An International Reliability and Optimization Study. Neurology 2010, 75, 1277-1284. [CrossRef]

163. Das, T.; Settecase, F.; Boulos, M.; Huynh, T.; D’Esterre, C.D.; Symons, S.P.; Zhang, L.; Aviv, R.I. Multimodal CT Provides Improved Performance for Lacunar Infarct Detection. Am. J. Neuroradiol. 2015, 36, 1069-1075. [CrossRef]

164. Benson, J.C.; Payabvash, S.; Mortazavi, S.; Zhang, L.; Salazar, P.; Hoffman, B.; Oswood, M.; McKinney, A.M. CT Perfusion in Acute Lacunar Stroke: Detection Capabilities Based on Infarct Location. Am. J. Neuroradiol. 2016, 37, 2239-2244. [CrossRef]

165. Cao, W.; Yassi, N.; Sharma, G.; Yan, B.; Desmond, P.M.; Davis, S.M.; Campbell, B.C.V. Diagnosing Acute Lacunar Infarction Using CT Perfusion. J. Clin. Neurosci. 2016, 29, 70-72. [CrossRef]

166. Garcia-Esperon, C.; Visser, M.; Churilov, L.; Miteff, F.; Bivard, A.; Lillicrap, T.; Levi, C.R.; Spratt, N.J.; Parsons, M.W. Role of Computed Tomography Perfusion in Identification of Acute Lacunar Stroke Syndromes. Stroke 2020, 339-343. [CrossRef]

167. Chapman, K.M.; Woolfenden, A.R.; Graeb, D.; Johnston, D.C.C.; Beckman, J.; Schulzer, M.; Teal, P.A. Intravenous Tissue Plasminogen Activator for Acute Ischemic Stroke. Stroke 2000, 31, 2920-2924. [CrossRef]

168. Pantoni, L.; Fierini, F.; Poggesi, A. Thrombolysis in Acute Stroke Patients with Cerebral Small Vessel Disease. Cerebrovasc. Dis. 2014, 37, 5-13. [CrossRef]

169. Matusevicius, M.; Paciaroni, M.; Caso, V.; Bottai, M.; Khurana, D.; de Bastos, M.; Martins, S.C.; Krespi, Y.; Cooray, C.; Toni, D.; et al. Outcome after Intravenous Thrombolysis in Patients with Acute Lacunar Stroke: An Observational Study Based on SITS International Registry and a Meta-Analysis. Int. J. Stroke 2019, 14, 878-886. [CrossRef]

170. Powers, W.J.; Rabinstein, A.A.; Ackerson, T.; Adeoye, O.M.; Bambakidis, N.C.; Becker, K.; Biller, J.; Brown, M.; Demaerschalk, B.M.; Hoh, B.; et al. 2018 Guidelines for the Early Management of Patients with Acute Ischemic Stroke: A Guideline for Healthcare Professionals from the American Heart Association/American Stroke Association. Stroke 2018, 49, e46-e99. [CrossRef]

171. Thomalla, G.; Boutitie, F.; Ma, H.; Koga, M.; Ringleb, P.; Schwamm, L.H.; Wu, O.; Bendszus, M.; Bladin, C.F.; Campbell, B.C.V.; et al. Intravenous Alteplase for Stroke with Unknown Time of Onset Guided by Advanced Imaging: Systematic Review and MetaAnalysis of Individual Patient Data. Lancet 2020, 396, 1574-1584. [CrossRef]

172. Barow, E.; Boutitie, F.; Cheng, B.; Cho, T.H.; Ebinger, M.; Endres, M.; Fiebach, J.B.; Fiehler, J.; Ford, I.; Galinovic, I.; et al. Functional Outcome of Intravenous Thrombolysis in Patients with Lacunar Infarcts in the Wake-up Trial. JAMA Neurol. 2019, 76, 641-649. [CrossRef] [PubMed]

173. Campbell, B.C.V.; Ma, H.; Ringleb, P.A.; Parsons, M.W.; Churilov, L.; Bendszus, M.; Levi, C.R.; Hsu, C.; Kleinig, T.J.; Fatar, M.; et al. Extending Thrombolysis to 4.5-9 h and Wake-up Stroke Using Perfusion Imaging: A Systematic Review and Meta-Analysis of Individual Patient Data. Lancet 2019, 394, 139-147. [CrossRef]

174. Vynckier, J.; Maamari, B.; Grunder, L.; Goeldlin, M.B.; Meinel, T.R.; Kaesmacher, J.; Hakim, A.; Arnold, M.; Gralla, J.; Seiffge, D.J.; et al. Early Neurologic Deterioration in Lacunar Stroke. Neurology 2021, 97, e1437-e1446. [CrossRef] [PubMed]

175. Kwok, C.S.; Shoamanesh, A.; Copley, H.C.; Myint, P.K.; Loke, Y.K.; Benavente, O.R. Efficacy of Antiplatelet Therapy in Secondary Prevention Following Lacunar Stroke: Pooled Analysis of Randomized Trials. Stroke 2015, 46, 1014-1023. [CrossRef] [PubMed]

176. Fergus, I.V. Effects of Clopidogrel Added to Aspirin in Patients with Recent Lacunar Stroke. N. Engl. J. Med. 2012, 367, 817-825. [CrossRef]

177. Wang, Y.; Wang, Y.; Zhao, X.; Liu, L.; Wang, D.; Wang, C.; Wang, C.; Li, H.; Meng, X.; Cui, L.; et al. Clopidogrel with Aspirin in Acute Minor Stroke or Transient Ischemic Attack. N. Engl. J. Med. 2013, 369, 11-19. [CrossRef]

178. Johnston, S.C.; Easton, J.D.; Farrant, M.; Barsan, W.; Conwit, R.A.; Elm, J.J.; Kim, A.S.; Lindblad, A.S.; Palesch, Y.Y. Clopidogrel and Aspirin in Acute Ischemic Stroke and High-Risk TIA. N. Engl. J. Med. 2018, 379, 215-225. [CrossRef]

179. Johnston, S.C.; Amarenco, P.; Denison, H.; Evans, S.R.; Himmelmann, A.; James, S.; Knutsson, M.; Ladenvall, P.; Molina, C.A.; Wang, Y. Ticagrelor and Aspirin or Aspirin Alone in Acute Ischemic Stroke or TIA. N. Engl. J. Med. 2020, 383, 207-217. [CrossRef]

180. Jing, J.; Meng, X.; Zhao, X.; Liu, L.; Wang, A.; Pan, Y.; Li, H.; Wang, D.; Johnston, S.C.; Wang, Y.; et al. Dual Antiplatelet Therapy in Transient Ischemic Attack and Minor Stroke with Different Infarction Patterns Subgroup Analysis of the CHANCE Randomized Clinical Trial. JAMA Neurol. 2018, 75, 711-719. [CrossRef]

181. Berberich, A.; Schneider, C.; Reiff, T.; Gumbinger, C.; Ringleb, P.A. Dual Antiplatelet Therapy Improves Functional Outcome in Patients With Progressive Lacunar Strokes. Stroke 2019, 50, 1007-1009. [CrossRef]

182. Takeuchi, M.; Miyashita, K.; Nakagawara, J.; Toyoda, K.; Todo, K.; Metoki, N.; Suzuki, R.; Manabe, Y.; Akaiwa, Y.; Yamamoto, Y.; et al. Analysis of Factors Associated with Progression and Long-Term Outcomes of Penetrating Artery Territory Infarction: A Retrospective Study. J. Stroke Cerebrovasc. Dis. 2016, 25, 1952-1959. [CrossRef]

183. Dawson, J.; Merwick, Á.; Webb, A.; Dennis, M.; Ferrari, J.; Fonseca, A.C. European Stroke Organisation Expedited Recommendation for the Use of Short-Term Dual Antiplatelet Therapy Early after Minor Stroke and High-Risk TIA. Eur. Stroke J. 2021, 6, CLXXXVII-CXCI. [CrossRef]

184. Wiśniewski, A.; Filipska, K. The Phenomenon of Clopidogrel High On-Treatment Platelet Reactivity in Ischemic Stroke Subjects: A Comprehensive Review. Int. J. Mol. Sci. 2020, 21, 6408. [CrossRef] 
185. Wiśniewski, A.; Sikora, J.; Karczmarska-Wódzka, A.; Sobczak, P. A Combination of Aspirin and Clopidogrel Predict More Favorable Dynamics of Platelet Reactivity versus Clopidogrel Alone in the Acute Phase of Minor Stroke. Healthcare 2021, 9, 628. [CrossRef]

186. McHutchison, C.; Blair, G.W.; Appleton, J.P.; Chappell, F.M.; Doubal, F.; Bath, P.M.; Wardlaw, J.M. Cilostazol for Secondary Prevention of Stroke and Cognitive Decline: Systematic Review and Meta-Analysis. Stroke 2020, 51, 2374-2385. [CrossRef]

187. Amarenco, P.; Benavente, O.; Goldstein, L.B.; Callahan, A.; Sillesen, H.; Hennerici, M.G.; Gilbert, S.; Rudolph, A.E.; Simunovic, L.; Zivin, J.A.; et al. Results of the Stroke Prevention by Aggressive Reduction in Cholesterol Levels (SPARCL) Trial by Stroke Subtypes. Stroke 2009, 40, 1405-1409. [CrossRef]

188. Ungvari, Z.; Toth, P.; Tarantini, S.; Prodan, C.I.; Sorond, F.; Merkely, B.; Csiszar, A. Hypertension-Induced Cognitive Impairment: From Pathophysiology to Public Health. Nat. Rev. Nephrol. 2021, 17, 639-654. [CrossRef]

189. Spence, J.D. Blood Pressure Gradients in the Brain: Their Importance to Understanding Pathogenesis of Cerebral Small Vessel Disease. Brain Sci. 2019, 9, 21. [CrossRef]

190. Liu, L.; Wang, Z.; Gong, L.; Zhang, Y.; Thijs, L.; Staessen, J.A.; Wang, J. Blood Pressure Reduction for the Secondary Prevention of Stroke: A Chinese Trial and a Systematic Review of the Literature. Hypertens. Res. 2009, 32, 1032-1040. [CrossRef]

191. Zhou, Z.; Xia, C.; Carcel, C.; Yoshimura, S.; Wang, X.; Delcourt, C.; Malavera, A.; Chen, X.; Mair, G.; Woodward, M.; et al. Intensive versus Guideline-Recommended Blood Pressure Reduction in Acute Lacunar Stroke with Intravenous Thrombolysis Therapy: The ENCHANTED Trial. Eur. J. Neurol. 2021, 28, 783-793. [CrossRef]

192. Deijle, I.A.; Van Schaik, S.M.; Van Wegen, E.E.H.; Weinstein, H.C.; Kwakkel, G.; Van Den Berg-Vos, R.M. Lifestyle Interventions to Prevent Cardiovascular Events after Stroke and Transient Ischemic Attack. Stroke 2017, 48, 174-179. [CrossRef]

193. Autenrieth, C.S.; Evenson, K.R.; Yatsuya, H.; Shahar, E.; Baggett, C.; Rosamond, W.D. Association between Physical Activity and Risk of Stroke Subtypes: The Atherosclerosis Risk in Communities Study. Neuroepidemiology 2013, 40, 109-116. [CrossRef]

194. Krawcyk, R.S.; Vinther, A.; Petersen, N.C.; Faber, J.; Hansen, R.H.; Rostrup, E.; Kruuse, C. Home-Based Aerobic Exercise in Patients with Lacunar Stroke: Design of the HITPALS Randomized Controlled Trial. Contemp. Clin. Trials Commun. 2019, 14, 100332. [CrossRef]

195. Han, S.W.; Lee, S.S.; Kim, S.H.; Lee, J.H.; Kim, G.S.; Kim, O.J.; Koh, I.S.; Lee, J.Y.; Suk, S.H.; Lee, S.I.; et al. Effect of Cilostazol in Acute Lacunar Infarction Based on Pulsatility Index of Transcranial Doppler (ECLIPse): A Multicenter, Randomized, Double-Blind, Placebo-Controlled Trial. Eur. Neurol. 2013, 69, 33-40. [CrossRef]

196. Stack, J.; Ryan, J.; McCarthy, G. Colchicine: New Insights to an Old Drug. Am. J. Ther. 2015, 22, e151-e157. [CrossRef] 